\title{
On Uncertainty Quantification in Hydrogeology and Hydrogeophysics
}

\author{
Niklas Linde ${ }^{\mathrm{a}, *}$, David Ginsbourger ${ }^{\mathrm{b}, \mathrm{c}}$, James Irving ${ }^{\mathrm{a}}$, Fabio Nobile ${ }^{\mathrm{d}}$, Arnaud \\ Doucet $^{\mathrm{e}}$ \\ ${ }^{a}$ Environmental Geophysics Group, Institute of Earth Sciences, University of Lausanne, \\ 1015 Lausanne, Switzerland \\ ${ }^{b}$ Uncertainty Quantification and Optimal Design group, Idiap Research Institute, Centre du \\ Parc, Rue Marconi 19, PO Box 592, 1920 Martigny, Switzerland \\ ${ }^{c}$ Institute of Mathematical Statistics and Actuarial Science, Department of Mathematics \\ and Statistics, University of Bern, Alpeneggstrasse 22, 3012 Bern, Switzerland \\ ${ }^{d}$ Calcul Scientifique et Quantification de l'Incertitude, Institute of Mathematics, Ecole \\ polytechnique fédérale de Lausanne, Station 8, CH 1015, Lausanne, Switzerland \\ ${ }^{e}$ Department of Statistics, Oxford University, 24-29 St Giles', Oxford, OX1 3LB, United \\ Kingdom
}

\begin{abstract}
Recent advances in sensor technologies, field methodologies, numerical modeling, and inversion approaches have contributed to unprecedented imaging of hydrogeological properties and detailed predictions at multiple temporal and spatial scales. Nevertheless, imaging results and predictions will always remain imprecise, which calls for appropriate uncertainty quantification (UQ). In this paper, we outline selected methodological developments together with pioneering UQ applications in hydrogeology and hydrogeophysics. The applied mathematics and statistics literature is not easy to penetrate and this review aims at helping hydrogeologists and hydrogeophysicists to identify suitable approaches for UQ that can be applied and further developed to their specific needs. To bypass the tremendous computational costs associated with forward UQ based on full-physics simulations, we discuss proxy-modeling strategies and multi-resolution (Multi-level Monte Carlo) methods. We consider Bayesian inversion for non-linear and non-Gaussian state-space problems and discuss how Sequential Monte Carlo may become a practical alternative. We also describe
\end{abstract}

\footnotetext{
* Corresponding author

Email address: niklas.linde@unil.ch (Niklas Linde)
}

Preprint submitted to Advances in Water Resources

October 11, 2017 
strategies to account for forward modeling errors in Bayesian inversion. Finally, we consider hydrogeophysical inversion, where petrophysical uncertainty is often ignored leading to overconfident parameter estimation. The high parameter and data dimensions encountered in hydrogeological and geophysical problems make UQ a complicated and important challenge that has only been partially addressed to date.

Keywords: Uncertainty quantification, hydrogeology, hydrogeophysics, inversion, proxy models, modeling errors, petrophysics

\section{Introduction}

The subsurface environment is highly heterogeneous and non-linear coupled processes take place at multiple spatial and temporal scales. Valuable information about subsurface structures and processes can be obtained from borehole 5 measurements, outcrops, laboratory analysis of field samples, and from geophysical and hydrogeological experiments; however, this information is largely incomplete. It is critical that basic scientific studies and management decisions for increasingly complex engineering challenges (e.g., enhanced geothermal systems, carbon capture and storage, nuclear waste repositories, aquifer storage and recovery, remediation of contaminated sites) account for this incompleteness in our system understanding. This enables us to consider the full range of possible future outcomes, to base scientific findings on solid grounds and to target future investigations. Nevertheless, uncertainty quantification (UQ) is highly challenging because it attempts to quantify what we do not know. For

15 example, it is extremely difficult to properly describe prior information about a hydrogeological system, to accurately quantify complex error characteristics in our data, and to quantify model errors caused by incomplete physical, chemical, and biological theories.

Eloquent arguments have been put forward to explain why numerical models in the Earth Sciences cannot be validated [1, 2]. These arguments are based on Popperian viewpoints [3] and on the recognition that natural subsurface systems 
are open and inherently under-sampled. This implies that UQ in the Earth Sciences can never be considered to be complete. Instead, it should be viewed as a partial assessment that is valid for a given set of prior assumptions, hypotheses, and simplifications. With this in mind, UQ in terms of probability distributions, often characterized in terms of probability density functions (pdfs), can still greatly help to make informed decisions regarding, for example, strategies for mitigating the effects of climate change, how to best exploit natural resources, how to minimize exposure to environmental pollutants, and how to protect environmental goods such as clean groundwater.

This review focuses on UQ in hydrogeology and hydrogeophysics. Using the term UQ, we refer both to (i) the forward UQ problem, namely how to characterize the distribution of output variables of interest (e.g., to determine the risk of contamination in a water supply well) given a distribution of input vari-

35 ables (e.g., subsurface material properties); and (ii) the solution of the Bayesian inverse UQ problem, whereby prior knowledge is merged with (noisy) observational data and numerical modeling in order to obtain a posterior distribution for the input variables. Note that it is beyond the scope of this work to make an exhaustive review of UQ or to present all existing and potential applications 40 in hydrogeology and hydrogeophysics. Rather, we try to connect a number of recent methodological advances in UQ with selected contemporary challenges in hydrogeology and hydrogeophysics. The mathematical development and the description of the methods are kept to a minimum and ample references are provided for further reading. We emphasize general methods that do not necfor this generality is a substantial increase in computational cost, which is reflected by the fact that more approximate approaches are presently favoured (e.g., Ensemble Kalman filters [4], quasi-static linear inversion [5]). Clearly, these approximate methods are not only used because they are comparatively 50 fast, but also because they have shown to produce useful and robust results in a wide range of application areas.

After introducing the main concepts and notations (section 2), we discuss 
the definition of prior distributions for spatially distributed parameter fields (section 3.1). This is followed by a discussion on the role of proxy models in forward UQ (section 3.2), after which we present how Multi-Level Monte Carlo and related techniques can be used within forward UQ to propagate prior uncertainties into quantities of interest (section 3.3). Next, we consider the Bayesian inverse problem where we examine likelihood functions (section 4.1) and discuss sampling approaches with an emphasis on particle methods (section 4.2). (section 5.1) and petrophysical-relationship uncertainty in hydrogeophysical inversions (section 5.2.

\section{Main concepts and notations}

In hydrogeology, it is often desirable to predict and characterize uncertainties on Quantities of Interest (QoI) given a set of inputs described by a multivariate parameter $\mathbf{u}$. Depending on the problem, $\mathbf{u}$ may refer to a vector, a field, a more general function, or combinations thereof; here, without loss of generality, we use the "field" as a generic term to denote $\mathbf{u}$. As an example, $\mathbf{u}$ may represent a permeability field and a contaminant source region, and the QoI may be the contaminant concentration in a water supply well at some future time. In this case, the forward model that links the two would typically be a numerical solver of the advection-dispersion equation for some set of (possibly uncertain) boundary and initial conditions. Herein, $\mathbf{u}$ is treated either as a discretized (finite-dimensional) or continuous (infinite-dimensional) object. This distinction

75 might seem superfluous at first because discretization is always needed at some stage when dealing with numerical forward models; however, considering an infinite-dimensional formalism can be highly relevant as discussed later.

A given QoI, denoted by $\mathbf{Q}$, is a function of the output from the considered solution map (in practice, the output of a numerical simulator), formalized as a deterministic function $\mathcal{R}: \mathbf{u} \mapsto \mathcal{R}(\mathbf{u})$ that is generally non-linear. Here, we use $\mathcal{Q}$ for the function mapping $\mathbf{u}$ to $\mathbf{Q}$. This function can be formulated as $\tilde{\mathcal{Q}} \circ \mathcal{R}$ 
for some function $\tilde{\mathcal{Q}}$ as $\mathbf{Q}$ is assumed to depend on $\mathbf{u}$ solely via $\mathcal{R}(\mathbf{u})$ so that $\mathbf{Q}=\mathcal{Q}(\mathbf{u})=\tilde{\mathcal{Q}}(\mathcal{R}(\mathbf{u}))$.

In essence, the probabilistic approach to forward UQ consists of endowing the considered set of $\mathbf{u}$ 's with a probability distribution $\mu_{0}$, and propagating this distribution to $\mathbf{Q}$ by using uncertainty-propagation techniques. The standard means of doing this, referred to as the basic Monte-Carlo method, consists of drawing a sample $\left\{\mathbf{u}_{1}, \ldots, \mathbf{u}_{N}\right\}$ from $\mu_{0}$, calculating the corresponding sample $\left\{\mathcal{Q}\left(\mathbf{u}_{1}\right), \ldots, \mathcal{Q}\left(\mathbf{u}_{N}\right)\right\}$, and empirically approximating expectations of functions of $\mathbf{Q}$ under the discrete probability distribution $\frac{1}{N} \sum_{i=1}^{N} \delta_{\mathcal{Q}\left(\mathbf{u}_{i}\right)}$.

Practical and theoretical work over the past decade has focused on how to best account for imperfect numerical modeling (see section 3.2), for instance via error models, and how to take advantage of multiple numerical models with different levels of fidelity and computation times (see section 3.3. Overall, propagating uncertainties in the inputs, accounting for imperfect numerical modeling, and addressing real-world problems using statistical procedures and numerical models are broadly considered as part of uncertainty propagation or forward $U Q$.

Inverse problems have played an important role in applied mathematics for more than a century and are of crucial importance in hydrogeology (e.g., [6, 7, 8 , ) and geophysics (e.g., [9, 10, 11]). The starting point when solving an inverse problem is to write the relation linking observed data $\mathbf{y}$ to model parameters $\mathbf{u}$

$$
\mathbf{y}=\mathcal{G}(\mathbf{u})+\epsilon,
$$

where the forward map $\mathcal{G}: \mathbf{u} \mapsto \mathcal{G}(\mathbf{u})$ can be viewed as the combination of a solution map $\mathcal{R}$ and an observation map $\mathcal{O}$ that returns $n \geq 1$ functionals of $\mathcal{R}(\mathbf{u})$ (typically linear forms, such as point-wise evaluations at specific locations and/or times), and $\boldsymbol{\epsilon}$ typically stands for observational noise. In simpler terms, $\mathcal{O}$ extracts from the output of the solution map the information that is needed to calculate the forward responses $\mathcal{G}(\mathbf{u})=\mathcal{O}(\mathcal{R}(\mathbf{u}))$, that are to be compared with the observed data $\mathbf{y}$.

For example, u may stand for lithological properties of an aquifer, with 
$\mathcal{R}$ returning the space-time evolution of contaminant concentration within this aquifer. The corresponding $\mathcal{O}$ could indicate concentrations at specific well locations and times, and the inverse problem would then consist of recovering the unknown lithology from noisy measurements $\mathbf{y}$ at these locations. In practice, $\mathcal{G}$ is the best possible numerical prediction of an experiment, but it is never a perfect map in a strict mathematical sense. This implies that virtually all $\mathcal{G}$ 's in the geosciences could be considered as proxy models (see section 3.2 ) and we use $\mathcal{G}$ herein when referring to high-fidelity forward simulations. While we do not explicitly consider $\boldsymbol{\epsilon}$ terms that incorporate model errors at this stage, the topic is implicitly tackled in forthcoming sections on likelihood functions and error modeling.

The inherent inaccuracies of forward solvers $\mathcal{G}$ have two origins. First, geological and physical heterogeneity are present at all scales, but numerical forward solvers can only handle heterogeneity up to a given spatial (e.g., model cell size) or spectral (e.g., truncation of spherical harmonics) resolution. The impact of limited resolution on simulation results depends strongly on the physics involved. For example, predicted gravimetric or groundwater-level responses will be comparatively insensitive, whereas seismic or ground penetrating radar (GPR) fullwaveform modeling or tracer transport simulation results may be highly sensitive [12. Second, considerable simplifications of the underlying physics are often made, even when using the most advanced simulation algorithms. The needed simplifications and their impacts are strongly problem dependent. For instance, gravimetric modeling can be performed using physical descriptions that are highly accurate, whereas GPR forward modeling typically does not account for the well-known frequency-dependence of subsurface electrical properties or the finite sizes of transmitter and receiver antennas [13. Furthermore, the accuracy of $\mathcal{G}$ for a given physical description and model domain depends also on the numerical schemes (e.g., in time) and equation solvers (e.g., iterative, direct) employed. Despite these simplifications, evaluating $\mathcal{G}(\mathbf{u})$ (i.e., solving the forward problem) often leads to significant computing times (e.g., [14, 15]), which limits the number of forward simulations that can be practically considered. 
In hydrogeology and geophysics, $\mathbf{u}$ is generally high-dimensional, $\mathcal{G}$ is costly to evaluate and non-linear, and the size of $\mathbf{y}$ is limited by data acquisition constraints. Bayesian inversion (the inverse UQ problem) provides a framework to make inferences on $\mathbf{u}$ from observations $\mathbf{y}$ by formulating and inferring the posterior distribution $\mu^{\mathrm{y}}$. Since analytical derivations of posterior distributions are generally intractable, Bayesian inverse problems call for Markov chain Monte Carlo (MCMC) and related sampling procedures (see section 4.2). Below, we first focus on the topic of defining the prior $\mu_{0}$ (i.e., a probabilistic description of model parameter values and their relations before considering the observed data); an essential component both in uncertainty propagation (forward UQ) and Bayesian inversion (inverse UQ).

\section{Prior distributions and forward UQ}

\subsection{Prior distributions on parameter fields}

Defining a prior distribution, $\mu_{0}$, for a spatial parameter field $\mathbf{u}$ is a challenging task. Since the advent of geostatistics, and notably the seminal works of Krige [16] and Matheron [17, a central approach underlying the prediction of spatially distributed variables has been to view the true but unknown field of interest as one realization of a random field (i.e., a random process with multivariate index space). In basic versions of kriging, no distributional assumptions on the field were made beyond the existence of moments. However, the Gaussian assumption delivers a way to express the simple-kriging equations in terms of conditional expectation and variance, thus allowing for conditional simulations [18, 19. With time, this initial Gaussian model was further developed to account for positivity (e.g., with log-Gaussian fields) and other constraints [20, 21]. Connections between kriging, Gaussian random fields, and Bayesian inference have been made notably in [22, 23, 24, 25, 11, 26]. This has led to a number of developments, for instance, hierarchical models that include distributions on hyperparameters describing Gaussian process models [27, 28. Throughout the 
also that the Gaussian-random-field terminology is equivalent to what is often referred to as multi-Gaussian in the geosciences.

In mathematics, Gaussian-related priors have been recently revived through their omnipresence in the blossoming field of UQ. Due to their favorable properties and well developed mathematical theory, Gaussian random fields, or equivalently Gaussian measures on function spaces [29], have been extensively used in the study of stochastic partial differential equations (PDEs) 30, 31] and PDEs with random coefficients (e.g., 32]). Recent contributions to the stochastic PDE approach to Gaussian-random-field modelling have highlighted its ability to cope with large data sets and to encode non-stationarity in a powerful way [33, 34, 35, 36]. Also, theoretical aspects of infinite-dimensional Bayesian inverse problems with Gaussian-random-field priors have been investigated [37, 38, 39], where $\mu_{0}$ is specified in terms of random series $\mathbf{u}=\phi_{0}+\sum_{j=1}^{+\infty} u_{j} \phi_{j}$, with $\phi_{j}$ denoting functions in a Banach space (i.e., a complete normed vector space) and $u_{j}$ Gaussian random coefficients. Non-Gaussian extensions (e.g., for uniformly distributed $u_{j}$ 's) have also been considered [40, 41, 42].

The impact of non-Gaussian property fields on stochastic forward simulations have been investigated (e.g., [43]) with results illustrating that covariances are insufficient to characterize geologically realistic subsurface properties. 190 To address this, multiple-point statistics (MPS) simulation has arisen as a new paradigm that has deeply influenced modern geostatistics [44, 45, 46, 47, 48]. Connections between MPS and Markov random fields [49, 50, texture synthesis developed for computer graphics purposes [51, and universal kriging [52] have been investigated. [53] studied the ability of MPS to reproduce statistical properties of a random field by averaging over a large number of MPS realizations obtained from a single training image. Exact statistical recovery was only shown to be possible when the training image was an "infinitely" large realization of a stationary and ergodic random field (i.e., statistical properties do not change in space and statistics can be recovered from one realization). The influence of 200 Gaussian-random-field and training-image-based priors on the solution of geophysical inverse problems was examined in [54]. It was found that complex 
prior information not only enhances the geological realism of posterior model realizations, but also renders the inference problem easier and faster to solve compared to the case of non-constraining priors. In field applications, the main challenge in applying MPS is how to obtain representative training images. For recent reviews on geologically realistic prior model definitions and inversion, we refer to [55, 56].

The process of choosing realistic and implementable prior distributions is a crucial yet rarely addressed topic that is often restricted to mean and covariance selection for Gaussian random fields or training image definition in MPS. In all instances, choices must be made that may dramatically influence forward UQ and the posterior distributions obtained through Bayesian inversion. Already for the Gaussian case, designing the covariance function (kernel) is a delicate task that implies a range of assumptions on the physical attributes for which one is inverting. For instance, the choice of a specific family of covariance function automatically defines the spatial regularity (smoothness class) of each realization drawn from the prior distribution and, hence, from the posterior distribution as well (see [57] for results in the Gaussian case and beyond). The impact of the prior is clearly shown in $[54$ who inverted the same synthetic data set using different prior models (Fig. 1). It is seen that the spatial statistics are largely determined by the prior model, while regions of predominantly high- or low velocities are determined by the data used in the inversion.

\subsection{Proxy models for forward $U Q$}

Proxy or surrogate models are often used when the full or high-fidelity forward response is too expensive to be systematically used in computations. They are commonly employed when a large number of forward simulations are required for UQ or sensitivity analysis applications. Proxy models can be grouped into two broad categories: lower-fidelity models and metamodels. Lower-fidelity proxies are typically physically-based; however, they contain less detail and therefore offer a less accurate, but cheaper-to-run, means of computing forward responses than their high-fidelity counterparts. Model simplifications are gen- 
erally made by (i) considering only some of the physics involved, either through approximations or by explicitly ignoring particular elements (e.g., [58]); (ii) reducing the numerical accuracy of the forward model response by, for example, coarsening the spatial discretization (e.g., [59]) or using model-order-reduction (MOR) approaches (e.g., [60]). In contrast, metamodels are usually not linked to the physics of the problem at hand. Instead, they are based on data-driven approximations of the forward model response using a relatively small number of high-fidelity simulation outputs. Methods that fall into the latter category notably include response surface modeling (RSM) (e.g., 61]), polynomial chaos expansion (PCE) (e.g., 62]), artificial neural networks (e.g., [63]), radial basis functions (e.g., 64]), and Gaussian process (GP) models 65, 66].

Hydrogeology has seen significant use of proxy models for forward UQ and sensitivity analysis. Being physically-based, lower-fidelity models have the advantage over metamodels in that they may better emulate the original response in unexplored regions of the input parameter space and are generally less susceptible to problems in high parameter dimensions (e.g., 67]). In this regard, [68, 69] employ simplified-physics proxies for subsurface flow and transport together with distance and kernel methods (e.g., 70]) in order to select, from a large number of permeability fields, a small subset of representative fields upon which to run high-fidelity forward simulations.

In terms of metamodels, many studies have focused on the application of PCE-based methods to hydrogeological problems (e.g., [71, 72]). Basically, a PCE represents the response of a complex system by a polynomial expansion with respect to the input random variables. When using PCEs, polynomials must be chosen that form an orthogonal basis with respect to the assumed probability distribution of input random variables. An important advantage of PCE over other metamodels is that it delivers polynomial approximations that are fast to evaluate and can lead to closed-form expressions (e.g., for Sobol' sensitivity indices) provided that the orthogonal polynomial basis functions are chosen accordingly [73]. Initial work was limited to low-dimensional problems because of the marked increase in the required number of $\mathrm{PCE}$ terms with the number 
of input parameters. However, recent applications involving sparse grids and truncated spectral expansions of the input random fields report successes with problems involving hundreds of model parameters. Nevertheless, the effectiveness of PCE techniques deteriorates when dealing with input random fields that are rough and/or have short correlation lengths. Hydrogeological applications of metamodeling with Gaussian process models include [74 that considered a hydrogeological transport problem. Here, the use of Gaussian process models were shown to outperform boosting regression trees and linear regression on most considered outputs. Another example is [75, in which a Gaussian process model incorporating proxy simulations and distance information was proposed for a sequential inversion problem where the candidate inputs were generated using MPS simulation.

\subsection{Forward UQ with Multi-level Monte Carlo}

Consider the forward problem of reliably computing the expectation of some quantity of interest $\mathbf{Q}$ involving the solution of the forward model, $\mathbf{Q}=\mathcal{Q}(\mathbf{u})$, where $\mathbf{u}$ is assumed random with prior distribution $\mu_{0}$ (hence $\mathbf{Q}$ is a random variable). Examples of QoIs could be tracer breakthrough curves or contaminant concentrations for an assumed prior distribution of lithological properties (e.g., porosity, permeability). In practice, approximations of $\mathcal{Q}(\mathbf{u})$ can only be obtained by numerical simulations that inevitably require discretization or physical simplifications (see section 2 ). We denote by $\mathcal{Q}_{\ell}(\mathbf{u})$ any such numerical solution, where $\ell$ denotes the resolution level. The latter may refer to the spatial grid discretization and/or time step increments used in the forward simulator, or any other type of model simplification.

In recent years, the so-called Multi Level Monte Carlo (MLMC) method has been established as a computationally efficient sampling method that builds upon the classical Monte Carlo technique. It was first proposed in [76] for applications in parametric integration, and then extended to weak approximations of stochastic differential equations in [77] together with a full complexity analysis. The idea behind MLMC is to introduce multiple levels $\ell=0, \ldots, L$ of increasing 
resolution (accuracy) with corresponding numerical solutions $\mathbf{Q}_{0}=\mathcal{Q}_{0}(\mathbf{u}), \mathbf{Q}_{1}=$ $\mathcal{Q}_{1}(\mathbf{u}), \ldots, \mathbf{Q}_{L}=\mathcal{Q}_{L}(\mathbf{u})$. While a classical Monte-Carlo approach would simply approximate the expected value of $\mathbf{Q}_{L}$ on a sufficiently high-resolution level $L$ using an ensemble-average over a sample of independent realizations from $\mu_{0}$, the MLMC method relies upon the simple observation that, by linearity of expectation,

$$
\mathbf{E}[\mathbf{Q}] \approx \mathbf{E}\left[\mathbf{Q}_{L}\right]=\sum_{\ell=1}^{L} \mathbf{E}\left[\mathbf{Q}_{\ell}-\mathbf{Q}_{\ell-1}\right]+\mathbf{E}\left[\mathbf{Q}_{0}\right],
$$

and computes each expectation in the sum by statistically independent Monte- 
by a Gaussian-random-field prior describing the logarithm of permeability. Using different quantities of interest and a pre-defined approximation error, they investigated the performance of MC, MLMC with a grid hierarchy of five levels, and an alternative MLMC approach based on a solver hierarchy using fast streamline-based and full reservoir-simulator predictions. With $\mathbf{Q}$ representing the mean saturation field at a given time, they found that MLMC with grid hierarchy and with solver hierarchy were 28.7 and 3.3 times faster than MC, respectively (Fig. 2). The authors argue that the solver-based hierarchy might be more practical when boundary conditions cannot be accurately defined with a coarse mesh. Combinations of MLMC techniques and metamodels based on sparse-grid PCE approximations have also been proposed [95] to further accelerate the computation of expectations in forward UQ problems with rough input permeability fields.

\section{Bayesian inversion}

It is well understood [96] that inverse problems are ill-posed unless the search space is drastically restricted. Standard deterministic inversion approaches proceed by penalizing a measure of model structure (e.g., relying on gradients, curvatures, or deviations from a reference model), thereby leading to a unique "regularized" solution. Deterministic approaches are popular because of their simplicity and the efficiency of the associated numerical methods. Although obtaining a unique solution is appealing, these methods do not provide a reliable assessment of uncertainty.

For a finite set of model parameters, a general formulation of the inverse problem is found in the work of [97, wherein the solution of the problem is described as the conjunction of two states of information: $(i)$ a density function describing the prior information about the system, including both the outputs of measurement instruments (i.e., the data) and prior assumptions about model parameter values; and (ii) a density function describing theoretical relationships between model parameters and data. This framework, which naturally accounts 
for forward modeling errors, makes it possible to solve the majority of non-linear inverse problems provided that appropriate density functions and the necessary computing resources are available.

Here we focus on the case when $\mathcal{G}$ is deterministic and we follow a classical Bayesian approach, which is extendable to infinite-dimensional model parameter spaces. This approach consists in combining a prior probability distribution $\mu_{0}$ of $\mathbf{u}$ with observed data in order to obtain the posterior distribution, $\mu^{\mathbf{y}}$. In $d$ dimensional cases where $\mu_{0}$ and the probability distribution $\nu_{0}$ of the error term $\epsilon$ have probability densities $\rho_{0}$ and $\rho$ with respect to some given measures (e.g., Lebesgue measures on $\mathbb{R}^{\ell}$ with $\ell=d, n$, respectively), one denotes by likelihood the function $\mathbf{u} \mapsto L(\mathbf{u} ; \mathbf{y}):=\rho(\mathbf{y}-\mathcal{G}(\mathbf{u}))$. Note that the likelihood is also often noted $L(\mathbf{u} \mid \mathbf{y})$, but should generally not be confused with the conditional density of $\mathbf{u}$ knowing $\mathbf{y}$. Assuming further that $Z:=\int_{\mathbf{R}^{d}} \rho(\mathbf{y}-\mathcal{G}(\mathbf{u})) \rho_{0}(\mathbf{u}) \mathrm{d} \mathbf{u}>0$ then $\mu^{\mathbf{y}}$ has the posterior density (Bayes' theorem)

$$
\rho^{\mathbf{y}}(\mathbf{u})=\frac{1}{Z} \rho(\mathbf{y}-\mathcal{G}(\mathbf{u})) \rho_{0}(\mathbf{u})=\frac{1}{Z} L(\mathbf{u} ; \mathbf{y}) \rho_{0}(\mathbf{u}),
$$

as recalled in 42 and generalized to the infinite-dimensional case as follows. Provided that the translate of $\nu_{0}$ by $\mathcal{G}(\mathbf{u}), \nu_{\mathbf{u}}$, possesses a density $\frac{\mathrm{d} \nu_{\mathbf{u}}}{\mathrm{d} \nu_{0}}(\mathbf{y})=$ $\exp (-\Phi(\mathbf{u} ; \mathbf{y}))$ with respect to $\nu_{0}$ for some function $\Phi$ referred to as potential, and assuming that $Z:=\int \exp (-\Phi(\mathbf{u} ; \mathbf{y})) \mathrm{d} \mu_{0}(\mathbf{u})>0$, then the posterior distribution $\mu^{\mathbf{y}}$ possesses a density with respect to $\mu_{0}$ with:

$$
\frac{\mathrm{d} \mu^{\mathbf{y}}}{\mathrm{d} \mu_{0}}(\mathbf{u})=\frac{1}{Z} \exp (-\Phi(\mathbf{u} ; \mathbf{y})) .
$$
bution via reweighting. Following [42], in the case where $\nu_{0}=\mathcal{N}(0, \Gamma)$ for some $n \times n$ invertible covariance matrix $\Gamma$, the potential function is

$$
\Phi(\mathbf{u} ; \mathbf{y})=\frac{1}{2}\left\|\Gamma^{-1 / 2}(\mathbf{y}-\mathcal{G}(\mathbf{u}))\right\|_{R^{n}}^{2}-\frac{1}{2}\left\|\Gamma^{-1 / 2} \mathbf{y}\right\|_{R^{n}}^{2} .
$$

Analytical formulations for density values and more particularly density ratios make it possible to apply the Metropolis-Hastings algorithm and to generalize it to infinite-dimensional settings. Quoting [42, it is expected that "formulating 
the theory and algorithms on the underlying infinite dimensional space [...] enables constructing algorithms which perform well under mesh refinement, since they are inherently well-defined in infinite dimensions."

\subsection{Likelihoods in geoscientific inverse problems}

Two important components that must be specified before inferring the posterior distribution $\mu^{\mathbf{y}}$ are the forward map $\mathcal{G}$ and the noise distribution $\nu_{0}$, which together determine the likelihood function $L(\cdot ; \mathbf{y})$ for the finite-dimensional case and/or the potential function $\Phi(\cdot ; \mathbf{y})$ for the infinite-dimensional case. These functions are used to evaluate how likely a given model realization is given the observed data and its noise characteristics. To allow for a large number of forward simulations (as needed for inverse-problem solving), it is often necessary to favor computational speed and make concessions in terms of simulation accuracy. The appropriate trade-off between time-consuming high-fidelity simulations and many fast, but approximate, solutions is problem dependent. Optimal determination of this trade-off is an important topic that we do not treat herein. Presently, the vast majority of Bayesian inversion studies in the geosciences implicitly assume that forward simulators are perfect and hence that modeling errors are negligible (i.e., only observational errors are considered). When acknowledged, the modeling errors are usually considered to be part of $\nu_{0}$ [98. Alternative approaches exist and formal ways to account for proxy errors are discussed in section 5.1. The latter often proceed by an adaptation of the likelihood function by correcting proxy simulations with an error model in order to obtain error-corrected simulations with a quality similar to that of highfidelity simulations (Fig. 3). There has been limited use of MLMC techniques in Bayesian inversion. The works [99, 100] have combined the multilevel idea with Metropolis-Hastings-type MCMC and, very recently, 101 applied the multilevel idea to Langevin dynamics to sample from a given distribution. An alternative approach to compute posterior expectations of QoIs, which does not resort to MCMC sampling but rather relies on standard MLMC or Quasi-Monte-Carlo integration, was proposed in [102]. 
Observational errors are most often treated as independent and identically distributed (iid) random variables with zero mean. These errors are typically considered to stem from Gaussian or Laplace distributions, partly because the corresponding likelihood functions have simple forms that are easy to manipulate. More advanced likelihood descriptions have been proposed. For example, [103] introduced and inverted for parameters describing a likelihood function with residual errors that are heteroscedastic and non-Gaussian with varying degrees of kurtosis and skewness. Hierarchical Bayes includes approaches in which parameters describing the likelihood function are considered uncertain.

It can be a very powerful approach to relax assumptions about parameter values describing the likelihood function, but it still requires a certain class of noise model to be selected for which the corresponding parameters are inferred. It is common to account for the combined effects of model and data errors in the likelihood function. For instance, 104 estimated hierarchical autoregressive error models that enable efficient handling of correlated errors at low computational costs (e.g., no need to invert the covariance matrix or compute its determinant in order to evaluate the likelihood function). In [105, the authors estimated a correlated error model and used it in the likelihood function to account for errors related to local heterogeneities close to GPR antennas. Using crosshole GPR data, 98] demonstrated how to practically sample a model-error distribution, which was found to be well described by a correlated multivariate Gaussian distribution. They demonstrated severe bias in the inferred posterior distributions when modeling errors were ignored.

\subsection{Sampling: Markov chain Monte Carlo and particle filters}

When performing Bayesian inference for complex statistical models, it is necessary to approximate numerically the resulting posterior distribution as it is typically intractable to compute analytically. For more than half a century, much effort has been placed on deriving sampling schemes for posterior distributions by relying on Markov chain Monte Carlo (MCMC) methods (see [106] and [107] for comprehensive reviews of the literature and [56] for the specific 
case of informed spatial priors). These schemes generally consist of sequential perturbations to candidate inputs $\mathbf{u}$ followed by either acceptance or rejection of the proposed perturbations with a probability that involves the likelihood ratio between the new and the old $\mathbf{u}$ and their prior probability ratio. Standard algorithms such as the Metropolis-Hastings algorithm and the Gibbs sampler have become very popular but they can be highly inefficient if the proposal distributions are not well-chosen and/or if the target (posterior) distribution exhibits complex patterns of dependence. A substantial research effort has thus been placed on making MCMC approaches more efficient, for instance, via parallel tempering 108, population MCMC 109] and/or through derivative-based perturbations with Metropolis-adjusted Langevin algorithms and Hamiltonian MCMC [110]. In infinite-dimensional settings, adaptations of MCMC schemes have been touched upon, notably in [11], and the links between performance and the spectral gap that controls the rate of exponential decay to $\mu^{\mathbf{y}}$ have been established in [112].

MCMC methods for Bayesian inverse problems are suitable when we are interested in inferring parameters, for example, a hidden (unobserved) static random field from data. However, there is also a wealth of data assimilation problems in hydrogeological and geophysical applications that can be recast 455 as statistical inference problems for non-linear and non-Gaussian state-space models [113, 4, 114, 115, 116, 117], with some of the published methods (e.g., [118, 119]) being applicable to complex prior information (e.g., MPS). We discuss below sampling techniques that have been developed in this context. These methods do not make any distributional assumptions on the prior distribution, but we highlight that it still remains to be investigated how they would perform within a MPS context.

Formally, a state-space model is defined by a discrete-time $\mathbf{R}^{n_{X}}$-valued hidden Markov process $\left(X_{t}\right)_{t \geq 1}$ such that $X_{1} \sim p_{\boldsymbol{\theta}}(\cdot)$ and $X_{t} \mid\left(X_{t-1}=\mathbf{x}\right) \sim$ $f_{\boldsymbol{\theta}}(\cdot \mid \mathbf{x})$ for $t \geq 2$ and we collect $\mathbf{R}^{n_{y}}$-valued observations $\left(Y_{t}\right)_{t \geq 1}$ which are 465 conditionally independent given $\left(X_{t}\right)_{t \geq 1}$ and distributed according to $Y_{t} \mid\left(X_{t}=\mathbf{x}\right) \sim$ $g_{\boldsymbol{\theta}}(\cdot \mid \mathbf{x})$. For example, if we assume that $Y_{t}=\phi\left(X_{t}\right)+\epsilon_{t}$ where $\epsilon_{t}$ is a mul- 
tivariate standard normal noise then $g(y \mid x)$ is the multivariate normal density of argument $y$, mean $\phi(x)$ and identity covariance. Here $\boldsymbol{\theta} \in \Theta$ denotes the parameters of the model. In the case of a static random field to be inferred, ${ }_{470} \boldsymbol{\theta}=\mathbf{u}$. When $\boldsymbol{\theta}$ is known, inference about $\left(X_{t}\right)_{t \geq 1}$ is referred to as state estimation. On-line inference (filtering) refers to sequential assimilation of the data as they become available. In batch/off-line inference (smoothing), the estimated states are also affected by the data acquired at later times. When $\boldsymbol{\theta}$ also needs to be estimated/calibrated from observations, this is referred to as parameter estimation and it can also be performed either on-line or off-line. In hydrogeology, $Y_{t}$ could represent salinity measurements within a coastal aquifer at some specific time, $X_{t}$ the corresponding salinity distribution throughout the same aquifer, and $\boldsymbol{\theta}$ an unknown hydraulic conductivity distribution and boundary conditions.

Standard MCMC methods can be used in this context, but it is often difficult to build efficient algorithms. In many fields such as computer vision, econometrics and robotics, particle methods, also known as Sequential Monte Carlo (SMC) methods, have emerged as the most successful class of techniques to address state estimation problems as they are easy to implement, suitable for both filtering and smoothing, admit parallel implementation and additionally provide asymptotically consistent state estimates. In its most generic form, SMC consists of initiating particles from an importance distribution at time zero, resampling them to ensure that they have the same weight, using the state associated with each particle to run a forward solver and analyze the resulting particle weight, and resampling until the particles at the new time have the same weight [120]. On- and off-line parameter estimation procedures building upon these state-estimation procedures have also been proposed; see [121 for a recent comprehensive review. An illustration of hydrogeophysical fully-coupled inversion using a particle filter [114] is given in Figure 4 . Other low-dimensional applications to hydrogeological and hydrogeophysical problems include [113, 122, 123, 115].

Nevertheless, SMC methods have not yet become prominent in hydrogeol- 
ogy. This is because $X_{t}$ often corresponds to a high-dimensional spatial field and the variance of SMC state estimates is typically exponential in the state dimension $n_{X}$ where routinely $n_{X}>10^{3}$. This problem is often referred to in the literature as the curse of dimensionality for particle methods [124]. Hence, practitioners rely on alternative approximation techniques such as the Ensemble Kalman filter (EnKF) [4, 116, 117. Empirically, the EnKF scales much better with $n_{X}$ than particle methods, but relies on potentially crude Gaussian approximations of the posterior distributions of interest. A non-standard particle method known as the equivalent weights particle filter has also been proposed and has shown empirical success in addressing high-dimensional data assimilation problems [125. However, it does not provide consistent state estimates and it is unclear how to control the error introduced by this scheme. The need for novel particle methods that can scale to high-dimensional settings has been recognized and there is a fast emerging literature addressing these problems in data assimilation and statistics [126, 127, 128, 129. A detailed theoretical analysis of such a scheme has been proposed in 130 where it was shown rigourously that it can overcome the curse of dimensionality. These methods provide asymptotically biased state and parameter estimates, the bias being controlled under suitable regularity assumptions, or consistent estimates whose mean square errors go to zero at a slower rate than the usual 1/N Monte Carlo rate [130, 131. The main idea behind these techniques is to ignore long-range dependencies when performing Bayes updates in a filtering procedure, an idea 520 borrowed from the ensemble Kalman filter literature where it is referred to as localization 4. The components of the state are partitioned into blocks and resampled using only the corresponding observations. Some of these methods are promising for high-dimensional hydrogeological and hydrogeophysical state and parameter estimation although several challenges remain to be addressed. 525 First, these methods introduce a non-homogeneous bias amongst state component estimates, which is damaging as $X_{t}$ often corresponds to a spatial field (e.g., salinity or soil moisture distribution) in hydrogeological applications [129]. Second, the smoothing and parameter estimation procedures developed in [131] 
cannot be applied when only forward simulation of $\left(X_{t}\right)_{t \geq 1}$ is feasible. Third,

$N$, the resulting rate of convergence is low and new efficient approaches are required.

An alternative class of particle-based techniques that provides consistent state and parameter estimates in high-dimensional settings are off-line procedures which build on particle MCMC methods, a class of MCMC methods relying on particle proposals introduced in [118]. For example, 132] presented a modification of the conditional SMC algorithm of [118 which performs empirically significantly better in high-dimensional settings by introducing positive correlation between particles [119]. 133] proposed a block Gibbs sampling scheme by updating the path of one state component at a time conditional on the other component paths. Although these techniques are not yet well-understood theoretically, they are highly promising. However, when they are used to perform parameter estimation, they alternate between updating $\boldsymbol{\theta}$ conditional to $\left(X_{t}\right)_{t \geq 1}$ and $\left(X_{t}\right)_{t \geq 1}$ conditional to $\boldsymbol{\theta}$. As the parameter and states are very often

\section{Selected challenges}

Below, we highlight two important topics for future research: namely, how to best account for modeling errors in hydrogeological Bayesian inversion (section 5.1 and for petrophysical errors in hydrogeophysical inversion (section 5.2). We describe existing work in these domains and possible paths forward.

\subsection{Accounting for modeling errors in Bayesian inverse problems}

Proxy models (section 3.2 are increasingly used in Bayesian inference for geoscientific problems, where it is not uncommon to require millions of for- 
ward model runs when dealing with high-dimensional parameter spaces. In [137. 138, for example, a 1D Richards equation is used to approximate 3D

be strongly non-Gaussian with characteristics that vary significantly over the input parameter space, meaning that the underlying assumptions are too simple and cannot be easily fixed by, for example, consideration of a more appropriate 
parametric distribution or formalized likelihood (e.g., [158, 103]). With regard to (ii), there is limited computational savings because each posterior sample acquired using two-stage MCMC must be tested with respect to the high-fidelity forward model.

In the field of statistics, one of the most influential works on model error is [159, whereby the discrepancy between the proxy and the high-fidelity simulation model is described by a GP. The approach is flexible as the parameters governing the GP are estimated as a part of the inversion procedure. Nevertheless, one issue with such an approach is that it is not guaranteed that the inferred model parameters and error model can be used for predictive forward modeling with different boundary conditions and forcing terms. Another key concern in the context of geoscience applications is model dimensionality. The vast majority of applications of [159] and its variants (e.g., [160, 161, 147, 162]) have focused on small numbers of data and low-dimensional parameter spaces. In contrast, spatially-distributed inverse problems in hydrogeology and geophysics may involve hundreds or thousands of data, often measured over both space and time and under different source conditions, and many thousands of unknowns. Nevertheless, when solving inverse problems over spatial domains, it is important to realize that the number of independent model parameters is typically much smaller than the number of grid elements on which the model realizations are mapped. This is indeed a major motivation for introducing spatial priors (Gaussian-random-field or based on MPS) as they help to make intractable inverse sampling problems tractable (see discussion in [56]).

In terms of practical applications, open questions include: (i) Can a GP model be used to effectively represent model discrepancy in problems where spatial and temporal correlations between model parameters and data are complex, the statistical nature of the modeling errors changes significantly over the input parameter space, and/or the model discrepancy is not smoothly varying? (ii) How can hydrogeological and geophysical data be transformed and/or spatially organized to enable appropriate representation of modeling errors using a GP model? (iii) How computationally burdensome does the approach of 


\subsection{Hydrogeophysics and uncertain petrophysical relationships}

Since the early 1990's [171, 172, 173, hydrogeology has seen an ever-increasing use (and acceptance) of geophysics. Geophysics offers non-invasive imaging of lithology and monitoring of mass transfer without the need for borehole access 
(even though such infrastructure is very helpful). It is well established that geophysical data offer complementary information to traditional hydrogeological data [174] (e.g., different sensitivity patterns and scales of investigation, no need to inject or pump water and solutes in the subsurface). Currently, there is a push towards so-called fully-coupled hydrogeological and geophysical modeling and inversion aiming at seamless integration of hydrogeological and geophysical data [175, 176]. In a fully-coupled approach, the hydrogeological model and its predicted states define, together with a petrophysical relationship, the geophysical model. Discrepancies between associated geophysical forward model predictions and observed data can then be used in the inversion to guide, possibly together with hydrogeological data, the update of the hydrogeological model parameters. This research field at the interface of hydrogeology and geophysics is often referred to as hydrogeophysics. Despite its promise, petrophysical relationships that link geophysical properties with hydrogeological properties and state variables are uncertain and we are not aware of hydrogeophysical inversion studies that fully account for this uncertainty. By referring to hydrogeophysical inversion, we exclude the extensive literature in hydrogeophysics on sequential approaches in which geophysical models are first obtained by inversion before these models are treated as "data" in a second stage to predict hydrological target variables given an uncertain petrophysical relationship and available hydrological data [172, 177]. The risk for strong bias when applying such approaches is well demonstrated [178. Ignoring petrophysical uncertainty in hydrogeophysical inversion leads to overconfident predictions and the risk that hydrogeological colleagues become disenchanted with geophysics [179]. In terms of methodology, the petrophysical relationship is the only major difference in hydrogeophysical inversion compared with classical hydrogeological inversion.

Before discussing the general non-linear case, we illustrate the strong impact of petrophysical uncertainty by considering the simple synthetic case of a linear forward model and a linear petrophysical relationship. For linear theory, a Gaussian-random-field prior model, Gaussian noise and petrophysical errors, one can propagate petrophysical uncertainties into the data covariance matrix 
and rely on well-known analytical solutions for the posterior mean and standard deviation [11]. Figure 6a is the true porosity field. Assuming a total of 729 first-arrival ground-penetrating radar travel times acquired for various source and receiver positions at the left and right side of the model domain (contaminated with $0.5 \mathrm{~ns}$ of uncorrelated Gaussian noise) and a perfect petrophysical relationship (black line in 6e) leads to the mean porosity field in Figure 60. The information content in the data is high and there is an important decrease in posterior porosity uncertainty (Fig. 6.) compared to the standard deviation of 0.04 in the prior model. Figure $6 \mathrm{~d}$ confirms that the resulting data covariance matrix is the conventional diagonal matrix. When accounting for uncorrelated petrophysical errors with strong (correlation coefficient of 0.85; Fig. 6e) and moderately strong (correlation coefficient of 0.59; Fig. 6;) petrophysical relationships, we find that the resulting mean porosity field is smoother (Figs. 6: and 6j), and that the posterior standard deviations are larger (Figs. 6s and 6k compared to the case of no petrophysical error. Importantly, the data covariance matrix that accounts for both data and petrophysical errors is no longer a diagonal matrix (Figs. 6h and 6 ). Clearly, petrophysical uncertainty decreases the information content of the geophysical data for hydrogeological inference and broadens the likelihood function (for the true model, the noise-contaminated data have a log-likelihood of -508 when there is no petrophysical errors, -944 for the strong petrophysical relationship and -1259 for the moderately strong petrophysical relationship). The impact of petrophysical errors is even stronger when considering spatial correlations (not shown). Unfortunately, the inference problem is much more complicated for the general non-linear case as discussed below.

Geophysical data (e.g., electrical resistances, electromagnetic transfer functions, waveform recordings) are related to subsurface physical properties (e.g., electrical conductivity, seismic wave speeds). In most applications, these properties represent hidden variables $\mathbf{v}$ of limited practical interest, while the underlying goals of geophysical surveys are often to infer state variables (e.g., temperature, pressure, water content, gas saturation) or lithological properties 
(e.g., porosity, permeability) of, for example, aquifers. For conciseness, we refer to all such target variables and properties as $\mathbf{u}$. When forward solvers take the hidden variables $\mathbf{v}$ rather than $\mathbf{u}$ as input, for example, via a non-linear geophysical "forward map" $\mathcal{G}_{V}: \mathbf{v} \mapsto \mathcal{G}_{V}(\mathbf{v})$, some knowledge of the petrophysical (rock physics) relationships that link $\mathbf{u}$ and $\mathbf{v}$ is required to infer $\mathbf{u}$ from geophysical observables $\mathbf{y}=\mathcal{G}_{V}(\mathbf{v})+\boldsymbol{\epsilon}$. These relationships are typically non-linear, uncertain, and non-stationary [180]. A possible description of such a relationship is

$$
\mathbf{v}=\mathcal{F}(\mathbf{u})+\boldsymbol{\epsilon}_{P}
$$
of $\epsilon_{P}$, we obtain a joint prior on $(\mathbf{u}, \mathbf{v})$ with density

$$
\rho_{\text {joint }, 0}(\mathbf{u}, \mathbf{v})=\rho(\mathbf{u}) \rho_{P}(\mathbf{v}-\mathcal{F}(\mathbf{u}))
$$

In geophysics, inference of the joint conditional distribution of $(\mathbf{u}, \mathbf{v})$ given geophysical data $\mathbf{y}$ is referred to as lithological tomography [183]. A recent tutorial [184] describes how to formulate Bayesian networks (using direct acyclic graphs) for arbitrarily complicated situations involving multiple data and parameter types, as well as a hierarchy of hidden variables. For simplicity, we focus our discussion on a single hidden variable $\mathbf{v}$. The standard approach (notably 
advocated by [184]) for posterior simulations of $\mathbf{u}$ consists in applying (variations of ) the Metropolis-Hastings algorithm to $(\mathbf{u}, \mathbf{v})$, where at each iteration the model perturbation consist in (i) drawing $\mathbf{u}$, and then (ii) drawing $\mathbf{v}$ conditionally on $\mathbf{u}$. Unfortunately, such a sampling strategy can be very inefficient when confronted with high parameter dimensions, large data sets with small errors $\boldsymbol{\epsilon}$, and uncertain petrophysical relationships. The main reason for this is that the likelihood $L_{V}(\mathbf{v} ; \mathbf{y})=\rho\left(\mathbf{y}-\mathcal{G}_{V}(\mathbf{v})\right)$ is very peaked, which implies that the geophysical data need to be fit in great detail even for cases when petrophysical uncertainty is significant (see discussion surrounding Fig. 6).

As alternatives, we suggest two approaches to directly sample from $\rho^{\mathbf{y}}(\mathbf{u})$ without needing to sample from $\rho_{\text {joint }}^{\mathbf{y}}(\mathbf{u}, \mathbf{v})$. The underlying motivation is to take advantage of the uncertainty of petrophysical relationships and work directly with approximations of $L_{U}(\mathbf{u} ; \mathbf{y})=\int L_{V}(\mathbf{v} ; \mathbf{y}) \rho_{P}(\mathbf{v}-\mathcal{F}(\mathbf{u})) \mathrm{d} \mathbf{v}$, which is expected to be less informative (i.e., less peaked) than $L_{V}(\mathbf{v} ; \mathbf{y})$. These approximations are needed as there are generally no closed-form expressions to evaluate $L_{U}(\mathbf{u} ; \mathbf{y})$.

The first approach builds on the pseudo-marginal MCMC method [185, 186] and the recent correlated pseudo-marginal method [187]. These methods are based on the remarkable property identified by [185] that a Metropolis-Hastings algorithm that uses a non-negative unbiased estimate $\tilde{L}_{U}(\mathbf{u} ; \mathbf{y})$ of $L_{U}(\mathbf{u} ; \mathbf{y})$ will sample the same target distribution as an ideal marginal Metropolis-Hastings algorithm that uses $L_{U}(\mathbf{u} ; \mathbf{y})$. Since the expression needed to evaluate $L_{U}(\mathbf{u} ; \mathbf{y})$ during MCMC sampling is unknown, it is convenient to estimate $\tilde{L}_{U}(\mathbf{u} ; \mathbf{y})$ by Monte Carlo averaging of $L_{V}(\cdot ; \mathbf{y})$ over samples of $\mathbf{v}$ conditional on $\mathbf{u}$. Clearly, $L_{V}(\cdot ; \mathbf{y})$ can be evaluated using standard likelihood expressions. The correlated pseudo-marginal method improves on the pseudo-marginal MCMC method by using correlated random samples to estimate the ratios between $\tilde{L}_{U}(\cdot ; \mathbf{y})$ values of the present and proposed models in the Metropolis-Hastings algorithm. This leads to lower variance estimates of the ratios, which results in significant performance improvements (e.g., two orders of magnitude).

The second approach relies on a linearized Gaussian approximation. A first- 
order expansion of $\mathcal{G}_{V}$ around $\mathcal{F}(\mathbf{u})$ delivers

$$
\mathcal{G}_{V}\left(\mathcal{F}(\mathbf{u})+\boldsymbol{\epsilon}_{P}\right) \approx \mathcal{G}_{V}(\mathcal{F}(\mathbf{u}))+\left\langle\nabla \mathcal{G}_{V}(\mathcal{F}(\mathbf{u})), \boldsymbol{\epsilon}_{P}\right\rangle .
$$

From there it is straightforward to derive the data covariance matrix of $\mathbf{y}$ given $\mathbf{u}$ by adding two distinct contributions: one related to the observational errors and the other one related to the petrophysical errors (after appropriate scaling with the Jacobian matrix). Assuming further Gaussian distributions for $\boldsymbol{\epsilon}_{P}$ and $\boldsymbol{\epsilon}$ leads to a completely determined Gaussian approximation for $L_{U}$. In essence, this is an extension of the linear analysis in Figure 6 to the weakly non-linear case. We expect this approach, which is similar to the so-called multivariate delta method [188, to be efficient when the Jacobian matrix is comparatively cheap to calculate. The accuracy of the method is expected to degrade with increasing non-linearity and degree of petrophysical uncertainty.

\section{Concluding remarks}

It is only recently that computational resources have enabled routine forward UQ and Bayesian sampling-based inversion for non-trivial problems involving high-parameter dimensions and complex prior distributions. In this review, we argue that (1) multi-resolution modeling using MLMC approaches is suitable for effective forward UQ given a distribution of material properties, while their role in inverse modeling remains to be explored; (2) general formulations of data assimilation problems based on particle methods (Sequential Monte Carlo) that are valid under strong non-linearity and non-Gaussianity are still underused in hydrogeology and geophysics and that more work is needed to enable accurate inference of posterior parameter distributions for such state-space models; (3) the use of low-fidelity (proxy) forward models are inevitable both for forward UQ and large-scale Bayesian inversion problems, while the question of how to quantify and efficiently account for modeling errors remains an important research topic; (4) that new approaches, such as the pseudo-marginal MCMC method, 
795 ical inversion and, thereby, to allow for proper weighting of hydrogeological and geophysical data in joint inversions and to avoid overly optimistic UQ. The high dimensionality and data rich environments encountered in modern hydrogeology and geophysics, together with complex spatial parameter relations, call for advanced mathematical and statistical methods that work well in high parameter and data dimensions. We hope that this review on selected topics on UQ will contribute in stimulating such research.

\section{Acknowledgments}

The first author would like to thank Susan Hubbard and the Lawrence Berke-

\section{References}

[1] L. F. Konikow, J. D. Bredehoeft, Ground-water models cannot be validated, Advances in Water Resources 15 (1) (1992) 75 - 83. doi:http: //dx.doi.org/10.1016/0309-1708(92)90033-X.

[2] N. Oreskes, K. Shrader-Frechette, K. Belitz, Verification, validation, and confirmation of numerical models in the earth sciences, Science 263 (5147) (1994) 641-646. doi:10.1126/science.263.5147.641

[3] A. Tarantola, Popper, Bayes and the inverse problem, Nature Physics 2 (8) (2006) 492-494. doi:10.1038/nphys375.

[4] G. Evensen, Data assimilation: The Ensemble Kalman Filter, Springer 820 Science \& Business Media, 2009. doi:10.1007/978-3-642-03711-5. 
[5] P. Kitanidis, Quasi-linear geostatistical theory for inversing, Water Resources Research 31 (10) (1995) 2411-2419. doi:\{10.1029/95WR01945\}.

[6] D. McLaughlin, L. R. Townley, A reassessment of the groundwater inverse problem, Water Resources Research 32 (5) (1996) 1131-1161. doi:10. 1029/96WR00160.

[7] J. Carrera, A. Alcolea, A. Medina, J. Hidalgo, L. J. Slooten, Inverse problem in hydrogeology, Hydrogeology Journal 13 (1) (2005) 206-222. doi:10.1007/s10040-004-0404-7

[8] H. Zhou, J. J. Gómez-Hernández, L. Li, Inverse methods in hydrogeology: Evolution and recent trends, Advances in Water Resources 63 (2014) 2237. doi:10.1016/j.advwatres.2013.10.014.

[9] W. Menke, Geophysical Data Analysis: Discrete Inverse Theory, Academic press, 2012.

[10] R. Parker, Geophysical Inverse Theory, Princeton University Press, Princeton, N.J., 1994.

[11] A. Tarantola, Inverse Problem Theory and Methods for Model Parameter Estimation, SIAM, 2005. doi:10.1137/1.9780898717921.

[12] M. Dentz, T. Le Borgne, A. Englert, B. Bijeljic, Mixing, spreading and reaction in heterogeneous media: A brief review, Journal of Contaminant Hydrology 120 (2011) 1-17. doi: j.jconhyd.2010.05.002

[13] A. Klotzsche, J. van der Kruk, N. Linde, J. Doetsch, H. Vereecken, 3 -D characterization of high-permeability zones in a gravel aquifer using 2-D crosshole GPR full-waveform inversion and waveguide detection, Geophysical Journal International 195 (2) (2013) 932-944. doi: $10.1093 / g j i / g g t 275$.

[14] A. Fichtner, Full Seismic Waveform Modelling and Inversion, Springer Science \& Business Media, 2010. 
[15] S. Geiger, S. Roberts, S. Matthäi, C. Zoppou, A. Burri, Combining finite element and finite volume methods for efficient multiphase flow simulations in highly heterogeneous and structurally complex geologic media, Geofluids 4 (4) (2004) 284-299.

[16] D. Krige, A statistical approach to some basic mine valuation problems on the Witwatersrand, Journal of the Chemical, Metallurgical and Mining Society of South Africa 52 (6) (1951) 119-139.

855 [17] G. Matheron, Principles of geostatistics, Economic Geology 58 (1963) 1246-1266.

[18] A. Journel, Geostatistics for conditional simulation of ore bodies, Economic Geology 69(5) (1974) 673-687.

[19] C. Lantuéjoul, Geostatistical Simulation-Models and Algorithms, Springer, 2002. doi:10.1007/978-3-662-04808-5.

q [20] N. Cressie, Statistics for Spatial Data, Wiley, 1993. doi:10.1002/ 9781119115151 .

[ [21] P. Diggle, P. J. Ribeiro, Model-based Geostatistics, Springer, 2007. doi: 10.1007/978-0-387-48536-2

[22] A. O'Hagan, Curve fitting and optimal design for prediction, Journal of the Royal Statistical Society. Series B (Methodological) 40 (1) (1978) 1-42.

[23] H. Omre, Bayesian kriging - merging observations and qualified guesses in kriging, Mathematical Geology 19 (1987) 25-39. doi:10.1007/ BF01275432,

870 [24] H. Omre, K. Halvorsen, The Bayesian bridge between simple and universal 1 kriging, Mathematical Geology 22 (7) (1989) 767-786. doi:10.1007/ BF00893321.

[25] M. S. Handcock, M. L. Stein, A Bayesian analysis of kriging, Technometrics 35 (4) (1993) 403-410. doi:10.2307/1270273. 
[26] T. M. Hansen, A. G. Journel, A. Tarantola, K. Mosegaard, Linear inverse gaussian theory and geostatistics, Geophysics 71 (6) (2006) R101.

[27] S. Banerjee, B. Carlin, A. Gelfand, Hierarchical Modeling and Analysis for Spatial Data, Monographs on Statistics and Applied Probability, Chapman and Hall/CRC, 2014.

[28] A. Gelman, J. B. Carlin, H. S. Stern, D. B. Dunson, A. Vehtari, D. B. Rubin, Bayesian Data Analysis, 3rd edition, Chapman \& Hall/CRC, 2013.

[29] B. Rajput, S. Cambanis, Gaussian processes and Gaussian measures, The Annals of Mathematical Statistics 43 (6) (1972) 1944-1952. doi:10.1214/ aoms/1177690865.

[30] M. Hairer, An introduction to stochastic pdes, lecture notes (2009). URL http://www . hairer . org/notes/SPDEs . pdf

[31] G. Da Prato, J. Zabczyk, Stochastic equations in infinite dimensions, 2nd Edition, Vol. 152 of Encyclopedia of Mathematics and its Applications, Cambridge University Press, Cambridge, 2014. doi:10.1017/ CB09781107295513.

[32] G. Lord, C. Powell, T. Shardlow, An introduction to computational stochastic PDEs, Cambridge Texts in Applied Mathematics, Cambridge University Press, New York, 2014. doi:10.1017/CB09781139017329.

[33] F. Lindgren, H. Rue, J. Lindström, An explicit link between gaussian fields and gaussian markov random fields: the stochastic partial differential equation approach, Journal of the Royal Statistical Society: Series B (Statistical Methodology) 73(4) (2011) 423-498.

[34] D. Simpson, F. Lindgren, H. Rue, In order to make spatial statistics computationally feasible, we need to forget about the covariance function, Environmetrics 23(1) (2012) 65-74. 
[35] R. Ingebrigtsen, F. Lindgren, I. Steinsland, S. Martino, Estimation of a non-stationary model for annual precipitation in southern Norway using replicates of the spatial field, Spatial Statistics 14(Part C) (2015) 338-364.

[36] G.-A. Fuglstad, F. Lindgren, D. Simpson, H. Rue, Exploring a new class of non-stationary spatial gaussian random fields with varying local anisotropy, Statistica Sinica 25(1) (2015) 115-133.

[37] A. Stuart, Inverse problems: A Bayesian perspective, Acta Numerica 19 (2010) 451-559. doi:10.1017/S0962492910000061.

[38] M. Dashti, A. Stuart, Uncertainty quantification and weak approximation of an elliptic inverse problem, SIAM Journal of Numerical Analysis 49 (2011) 2524-2542. doi:10.1137/100814664.

[39] P. Conrad, M. Girolami, A. Sarkka, S. Stuart, K. Zygalakis, Statistical analysis of differential equations: introducing probability measures on 『 numerical solutions, Statistics and Computing (2016) 1-18doi:10.1007/ s11222-016-9671-0

[40] V. H. Hoang, C. Schwab, N-term Wiener Chaos Approximation Rates for elliptic PDEs with lognormal Gaussian random inputs, Mathematical

口 Models and Methods in Applied Sciences 24(4) (2014) 797-826. doi: 10.1142/S0218202513500681.

[41] F. Kuo, C. Schwab, I. Sloan, Multi-level quasi-Monte Carlo finite element methods for a class of elliptic PDEs with random coefficients, Foundations of Computational Mathematics 15(2) (2015) 411449. doi: $10.1007 / \mathrm{s} 10208-014-9237-5$.

[42] M. Dashti, A. M. Stuart, The Bayesian approach to inverse problems, lecture notes to appear in Handbook of Uncertainty Quantification, Editors R. Ghanem, D. Higdon and H. Owhadi, Springer, 2017. arXiv:1302.6989. 
[43] Y. Rubin, A. Journel, Simulation of non-Gaussian space random functions for modeling transport in groundwater, Water Resources Research 27(7) (1991) 1711-1721. doi:10.1029/91WR00838.

[44] F. B. Guardiano, R. M. Srivastava, Multivariate geostatistics: Beyond bivariate moments, Springer Netherlands, Dordrecht, 1993, pp. 133-144. doi:10.1007/978-94-011-1739-5_12

[45] S. Strebelle, Conditional simulation of complex geological structures using multiple-point statistics, Mathematical Geology 34(1) (2002) 1-21. doi: 10.1023/A:1014009426274

[46] G. B. Arpat, J. Caers, Conditional simulation with patterns, Mathematical Geology 39(2) (2007) 177203. doi:10.1007/s11004-006-9075-3.

q [47] L. Y. Hu, T. Chugunova, Multiple-point geostatistics for modeling subsurface heterogeneity: A comprehensive review, Water Resources Research 44 (11) (2008) W11413, w11413. doi:10.1029/2008WR006993. URL http://dx.doi .org/10.1029/2008WR006993

[48] G. Mariéthoz, J. Caers, Multiple-Point Geostatistics: Stochastic Modeling with Training Images, John Wiley \& Sons, Ltd, 2014. doi:10.1002/ 9781118662953 .

[49] R. Dimitrakopoulos, H. Mustapha, E. Gloaguen, High-order statistics of spatial random fields: Exploring spatial cumulants for modeling complex non-gaussian and non-linear phenomena, Mathematical Geosciences 42(1) (2010) 65-99. doi:10.1007/s11004-009-9258-9.

[50] M. Stien, O. Kolbjørnsen, Facies modeling using a Markov mesh model 950 s specification, Mathematical Geosciences 43(6) (2011) 611-624. doi:10. 1007/s11004-011-9350-9.

[51] G. Mariéthoz, S. Lefebvre, Bridges between multiple-point geostatistics and texture synthesis: Review and guidelines for future research, Computers \& Geosciences 66 (2014) 66-80. doi:10.1016/j . cageo.2014.01.001. 
[52] L. Li, T. Romary, J. Caers, Universal kriging with training images, Spatial Statistics 14(C) (2015) 240-268. doi:10.1016/j.spasta.2015.04.004.

[53] X. Emery, C. Lantuéjoul, Can a training image be a substitute for a random field model?, Mathematical Geosciences 46 (2) (2014) 133-147. doi:10.1007/s11004-013-9492-z

[54] T. Hansen, K. Cordua, K. Mosegaard, Inverse problems with non-trivial priors: efficient solution through sequential Gibbs sampling, Computational Geosciences 16 (2012) 593611. doi:10.1007/s10596-011-9271-1

[55] N. Linde, P. Renard, T. Mukerji, J. Caers, Geological realism in hydrogeological and geophysical inverse modeling: A review, Advances in Water Resources 86 (2015) 86-101. doi:10.1016/j.advwatres.2015.09.019.

[56] T. M. Hansen, K. S. Cordua, A. Zunino, K. Mosegaard, Probabilistic integration of geo-information, in: N. L. M. Moorkamp, P. G. Lelivre, A. Khan (Eds.), Integrated Imaging of the Earth: Theory and Applications, John Wiley \& Sons, Inc, 2016, Ch. 5, pp. 93-116.

[57] M. Scheuerer, Regularity of the sample paths of a general second order random field, Stochastic Processes and their Applications 120 (2010) 18791897.

[58] L. Josset, D. Ginsbourger, I. Lunati, Functional error modeling for uncertainty quantification in hydrogeology, Water Resources Research 51(2) (2015) 1050-1068. doi:10.1002/2014WR016028.

[59] S. Arridge, J. Kaipio, V. Kolehmainen, M. Schweiger, E. Somersalo, T. Tarvainen, M. Vauhkonen, Approximation errors and model reduction with an application in optical diffusion tomography, Inverse Problems 22 (1) (2006) 175-195. doi:10.1088/0266-5611/22/1/010.

[60] X. Liu, Q. Zhou, J. Birkholzer, W. A. Illman, Geostatistical reduced-order models in underdetermined inverse problems, Water Resources Research 49 (10) (2013) 6587-6600. doi:10.1002/wrcr. 20489 
[61] R. Myers, D. Montgomery, C. Anderson-Cook, Response Surface Methodology: Process and Product Optimization Using Designed Experiments, Wiley, 2016.

[62] Y. Marzouk, D. Xiu, A stochastic collocation approach to Bayesian inference in inverse problems, Communications in Computational Physics 6 (2009) 826-847. doi:10.4208/cicp.2009.v6.p826.

[63] S.-T. Khu, M. Werner, Reduction of Monte-Carlo simulation runs for uncertainty estimation in hydrological modelling, Hydrology and Earth System Sciences 7 (5) (2003) 680-692. doi:10.5194/hess-7-680-2003.

[64] R. G. Regis, C. A. Shoemaker, A stochastic radial basis function method for the global optimization of expensive functions, INFORMS Journal on Computing 19 (4) (2007) 497-509. doi:10.1287/ijoc.1060.0182.

${ }_{995}^{6}$ [65] T. Santner, B. Williams, W. Notz, The Design and Analysis of Computer Experiments, Springer, New York, 2003.

[66] C. Rasmussen, C. Williams, Gaussian Processes for Machine Learning, the MIT Press, 2006. doi:10.1007/978-3-540-28650-9_4.

[67] S. Razavi, B. A. Tolson, D. H. Burn, Review of surrogate modeling in 1000 \ater resources, Water Resources Research 48 (7) (2012) W07401. doi: 10.1029/2011WR011527.

[68] C. Scheidt, J. Caers, Representing spatial uncertainty using distances and 口 kernels, Mathematical Geosciences 41 (4) (2009) 397-419. doi:10.1007/ s11004-008-9186-0

[69] L. Josset, I. Lunati, Local and global error models to improve uncertainty quantification, Mathematical Geosciences 45 (5) (2013) 601-620. doi: $10.1007 / \mathrm{s} 11004-013-9471-4$

[70] T. Hastie, R. Tibshirani, J. Friedman, The Elements of Statistical Learning, Springer, 2001. 
[74] A. Marrel, B. Iooss, F. Van Dorpe, E. Volkova, An efficient methodology for modeling complex computer codes with Gaussian processes, Computational Statistics \& Data Analysis 52 (2008) 4731-4744. doi: $10.1016 / j . c s d a .2008 .03 .026$.

[75] D. Ginsbourger, B. Rosspopoff, G. Pirot, N. Durrande, P. Renard, Distance-based kriging relying on proxy simulations for inverse conditioning, Advances in Water Resources 52 (2013) 275-291.

[76] S. Heinrich, Multilevel Monte Carlo methods, in: Large-Scale Scientific Computing, Vol. 2179 of Lecture Notes in Computer Science, Springer Berlin Heidelberg, 2001, pp. 58-67. doi:10.1007/978-3-642-41095-6_ 4 .

[77] M. B. Giles, Multilevel Monte Carlo path simulation, Operations Research 56 (3) (2008) 607-617. doi:10.1287/opre.1070.0496 
[78] A. Barth, C. Schwab, N. Zollinger, Multi-level Monte Carlo finite element method for elliptic PDEs with stochastic coefficients, Numerische Mathematik 119 (2011) 123-161. doi:10.1007/s00211-011-0377-0.

[79] A. Barth, A. Lang, C. Schwab, Multilevel Monte Carlo method for parabolic stochastic partial differential equations, BIT Numerical Mathematics 53 (1) (2013) 3-27. doi:10.1007/s10543-012-0401-5.

[80] J. Charrier, R. Scheichl, A. Teckentrup, Finite element error analysis of elliptic PDEs with random coefficients and its application to multilevel Monte Carlo methods, SIAM Journal on Numerical Analysis 51 (1) (2013) 322-352. doi:10.1137/110853054.

[81] K. Cliffe, M. Giles, R. Scheichl, A. Teckentrup, Multilevel Monte Carlo methods and applications to elliptic PDEs with random coefficients, Computing and Visualization in Science 14 (1) (2011) 3-15. doi:10.1007/ s00791-011-0160-x.

[82] S. Mishra, C. Schwab, J. Sukys, Multi-level Monte Carlo finite volume methods for nonlinear systems of conservation laws in multi-dimensions, (1) Journal of Computational Physics 231 (8) (2012) 3365-3388. doi:10. $1016 / j \cdot j c p .2012 .01 .011$

[83] A. L. Teckentrup, R. Scheichl, M. B. Giles, E. Ullmann, Further analysis of multilevel Monte Carlo methods for elliptic PDEs with random coefficients, Numerische Mathematik 125 (3) (2013) 569-600. doi: $10.1007 / \mathrm{s} 00211-013-0546-4$

[84] H. Harbrecht, M. Peters, M. Siebenmorgen, On multilevel quadrature for elliptic stochastic partial differential equations, in: Sparse Grids and Applications, Vol. 88 of Lecture Notes in Computational Sci口 ence and Engineering, Springer, 2013, pp. 161-179. doi:10.1007/ 978-3-642-31703-3_8 
[89] A.-L. Haji-Ali, F. Nobile, R. Tempone, Multi index Monte Carlo: when sparsity meets sampling, Numerische Mathematik 132 (4) (2016) 767-806.

[90] A.-L. Haji-Ali, F. Nobile, L. Tamellini, R. Tempone, Multi-index stochastic collocation convergence rates for random PDEs with parametric regularity, Foundations of Computational Mathematics 16 (2016) 1555-1605. doi:10.1007/s10208-016-9327-7

[92] S. Mishra, C. Schwab, J. Sukys, Multi-level Monte Carlo finite volume methods for shallow water equations with uncertain topography in multidimensions, SIAM Journal on Scientific Computing 34 (6) (2012) 761-784. doi:10.1137/110857295. 
[93] F. Müller, P. Jenny, D. Meyer, Multilevel Monte Carlo for two phase flow and Buckley-Leverett transport in random heterogeneous porous media, Journal of Computational Physics 250 (2013) 685-702. doi:10.1016/j . jcp.2013.03.023.

[94] F. Müller, D. W. Meyer, P. Jenny, Solver-based vs. grid-based multilevel Monte Carlo for two phase flow and transport in random heterogeneous porous media, Journal of Computational Physics 268 (2014) 39 - 50. doi: http://dx.doi.org/10.1016/j.jcp.2014.02.047

[95] F. Nobile, F. Tesei, A multi level Monte Carlo method with control variate for elliptic PDEs with log-normal coefficients, Stochastics and Partial Differential Equations: Analysis and Computations 3 (3) (2015) 398-444. doi:10.1007/s40072-015-0055-9

[100] V. Hoang, C. Schwab, A. Stuart, Complexity analysis of accelerated MCMC methods for Bayesian inversion, Inverse Problems 29 (8) (2013) 085010. doi:10.1088/0266-5611/29/8/085010. 
1120

[101] M. Giles, T. Nagapetyan, L. Szpruch, S. Vollmer, K. Zygalakis, Multilevel Monte Carlo for scalable Bayesian computations, arXiv:1609.06144.

[102] R. Scheichl, A. Stuart, A. Teckentrup, Quasi-Monte Carlo and multilevel Monte Carlo methods for computing posterior expectations in elliptic inverse problems, arXiv:1602.04704 (2016).

[103] G. Schoups, J. A. Vrugt, A formal likelihood function for parameter and predictive inference of hydrologic models with correlated, heteroscedastic, and non-Gaussian errors, Water Resources Research 46 (10) (2010) W10531. doi:10.1029/2009WR008933.

[104] J. Dettmer, S. Molnar, G. Steininger, S. E. Dosso, J. F. Cassidy, Transdimensional inversion of microtremor array dispersion data with hierarchical autoregressive error models, Geophysical Journal International 188 (2) (2012) 719-734. doi:10.1111/j.1365-246X.2011.05302.x.

[105] K. S. Cordua, L. Nielsen, M. C. Looms, T. M. Hansen, A. Binley, Quantifying the influence of static-like errors in least-squares-based inversion and sequential simulation of cross-borehole ground penetrating radar data, Journal of Applied Geophysics 68 (1) (2009) 71-84. doi: $10.1016 / \mathrm{j} \cdot \mathrm{j}$ appgeo.2008.12.002

[106] J. Liu, Monte Carlo Strategies in Scientific Computing, Springer, 2008. doi: 10.1007/978-0-387-76371-2.

[107] C. Robert, G. Casella, Monte Carlo Statistical Methods, Springer, 2013. doi:10.1007/978-1-4757-4145-2.

[108] D. Earl, M. Deem, Parallel tempering: Theory, applications, and new perspectives, Physical Chemistry Chemical Physics 7 (2005) 3910-3916. doi:10.1039/b509983h.

[109] C. J. F. Ter Braak, A Markov Chain Monte Carlo version of the genetic algorithm differential evolution: easy Bayesian computing for real 
parameter spaces, Statistics and Computing 16 (3) (2006) 239-249. doi: $\{10.1007 / \mathrm{s} 11222-006-8769-1\}$.

[110] R. Neal, Mcmc using hamiltonian dynamics, Handbook of Markov Chain Monte Carlo (2011) 113-162.

[111] S. L. Cotter, G. O. Roberts, A. M. Stuart, D. White, MCMC methods for functions: Modifying old algorithms to make them faster, Statistical Science 28(3) (2013) 424-446. doi:10.1214/13-STS421.

[112] M. Hairer, A. Stuart, S. Vollmer, Spectral gaps for a Metropolis-Hastings algorithm in infinite dimensions, The Annals of Applied Probability 24(6) (2014) 2455-2490. doi:10.1214/13-AAP982.

[113] S.-Y. Chang, T. Chowhan, S. Latif, State and parameter estimation with an SIR particle filter in a three-dimensional groundwater pollutant transport model, Journal of Environmental Engineering 138 (11) (2012) 11141121. doi:10.1061/(ASCE)EE.1943-7870.0000584

[114] G. Manoli, M. Rossi, D. Pasetto, R. Deiana, S. Ferraris, G. Cassiani, M. Putti, An iterative particle filter approach for coupled hydrogeophysical inversion of a controlled infiltration experiment, Journal of Computational Physics 283 (2015) 37-51. doi:10.1016/j·jcp.2014.11. 035 .

[115] C. Montzka, H. Moradkhani, L. Weihermüller, H.-J. Hendricks Franssen, M. Canty, H. Vereecken, Hydraulic parameter estimation by remotelysensed top soil moisture observations with the particle filter, Journal of Hydrology 399 (3) (2011) 410-421. doi:10.1016/j.jhydrol.2011.01. 020.

[116] D. S. Oliver, Y. Chen, Recent progress on reservoir history matching: a review, Computational Geosciences 15 (1) (2011) 185-221. doi:10.1007/ s10596-010-9194-2. 
[117] A. Schöniger, W. Nowak, H.-J. Hendricks Franssen, Parameter estima-

[123] D. Pasetto, M. Camporese, M. Putti, Ensemble Kalman filter versus par-

[124] T. Bengtsson, P. Bickel, B. Li, et al., Curse-of-dimensionality revisited: Collapse of the particle filter in very large scale systems, in: tion by ensemble Kalman filters with transformed data: Approach and application to hydraulic tomography, Water Resources Research 48 (4). doi:10.1029/2011WR010462.

[118] C. Andrieu, A. Doucet, R. Holenstein, Particle Markov chain Monte Carlo methods, Journal of the Royal Statistical Society: Series B (Statistical Methodology) 72 (3) (2010) 269-342. doi:10.1111/j.1467-9868.2009. $00736 . x$.

[119] A. Finke, A. Doucet, A. M. Johansen, On embedded hidden Markov models and particle Markov chain Monte Carlo methods, arXiv preprint arXiv:1610.08962.

[120] A. Doucet, A. M. Johansen, A tutorial on particle filtering and smoothing: fifteen years later (2011).

[121] N. Kantas, A. Doucet, S. Singh, J. Maciejowski, N. Chopin, et al., On particle methods for parameter estimation in state-space models, Statistical Science 30 (3) (2015) 328-351. doi:10.1214/14-STS511.

[122] J. Rings, J. Huisman, H. Vereecken, Coupled hydrogeophysical parameter estimation using a sequential Bayesian approach, Hydrology and Earth System Sciences 14 (3) (2010) 545-556. doi:10.5194/ hess-14-545-2010. ticle filter for a physically-based coupled surface-subsurface model, Advances in Water Resources 47 (2012) 1 - 13. doi:http://dx.doi.org/ 10.1016/j.advwatres.2012.06.009. Probability and statistics: Essays in honor of David A. Freedman, In- 
stitute of Mathematical Statistics, 2008, pp. 316-334. doi:10.1214/ 193940307000000518

[125] M. Ades, P. Van Leeuwen, An exploration of the equivalent weights particle filter, Quarterly Journal of the Royal Meteorological Society 139 (672) (2013) 820-840.

[126] S. Penny, T. Miyoshi, A local particle filter for high dimensional geophysical systems, Nonlinear Processes in Geophysics Discussions 2 (2015) 1631-1658.

[127] J. Poterjoy, A localized particle filter for high-dimensional nonlinear systems, Monthly Weather Review 144 (1) (2016) 59-76.

[128] J. Poterjoy, J. L. Anderson, Efficient assimilation of simulated observations in a high-dimensional geophysical system using a localized particle filter, Monthly Weather Review 144 (5) (2016) 2007-2020.

[129] S. Robert, H.-R. Künsch, Localization in High-Dimensional Monte Carlo Filtering, arXiv preprint arXiv:1610.03701.

[130] P. Rebeschini, R. Van Handel, Can local particle filters beat the curse of dimensionality?, The Annals of Applied Probability 25 (5) (2015) 28092866. doi:10.1214/14-AAP1061.

[131] A. Finke, S. Singh, Approximate smoothing and parameter estimation in high-dimensional state-space models, arXiv preprint arXiv:1606.08650.

[132] A. Y. Shestopaloff, R. Neal, Sampling latent states for high-dimensional non-linear state space models with the embedded HMM method, arXiv preprint arXiv:1602.06030.

[133] J. Murphy, S. J. Godsill, Blocked particle Gibbs schemes for high dimensional interacting systems, IEEE Journal of Selected Topics in Signal Processing 10 (2) (2016) 328-342. doi:10.1109/JSTSP.2015.2509940. 
[134] A. Doucet, M. K. Pitt, G. Deligiannidis, R. Kohn, Efficient implementation of Markov chain Monte Carlo when using an unbiased likelihood estimator, Biometrika 102 (2) (2015) 295-313. doi:10.1093/biomet/asu075

[140] A. O'Sullivan, M. Christie, Error models for reducing history match 口

[141] S. Balakrishnan, A. Roy, M. G. Ierapetritou, G. P. Flach, P. G. Geor1250

[135] G. Deligiannidis, A. Doucet, M. K. Pitt, The correlated pseudo-marginal method, arXiv preprint arXiv:1511.04992.

[136] P. E. Jacob, F. Lindsten, T. B. Schön, Coupling of particle filters, arXiv preprint arXiv:1606.01156.

[137] M. C. Looms, A. Binley, K. H. Jensen, L. Nielsen, Identifying unsaturated hydraulic parameters using an integrated data fusion approach on crossborehole geophysical data, Vadose Zone Journal 7(1) (2008) 238-248. doi: $10.2136 / \mathrm{vzj} 2007.0087$.

[138] M. Scholer, J. Irving, M. C. Looms, L. Nielsen, K. Holliger, Bayesian Markov-Chain-Monte-Carlo inversion of time-lapse crosshole GPR data to characterize the vadose zone at the Arrenaes site, Denmark, Vadose Zone Journal 11 (4). doi:10.2136/vzj2011.0153.

[139] P. Dostert, Y. Efendiev, B. Mohanty, Efficient uncertainty quantification techniques in inverse problems for Richards equation using coarse-scale simulation models, Advances in Water Resources 32 (3) (2009) 329-339. doi:10.1016/j.advwatres.2008.11.009

bias, Computational Geosciences 9 (2-3) (2005) 125-153. doi:10.1007/ s10596-006-9027-5 gopoulos, Uncertainty reduction and characterization for complex environmental fate and transport models: An empirical Bayesian framework incorporating the stochastic response surface method, Water Resources Research 39 (12). doi:10.1029/2002WR001810. 
[142] E. Laloy, B. Rogiers, J. A. Vrugt, D. Mallants, J. Diederik, Efficient posterior exploration of a high-dimensional groundwater model from twostage Markov chain Monte Carlo simulation and polynomial chaos expansion, Water Resources Research 49 (5) (2013) 2664-2682. doi: $10.1002 /$ wrcr. 20226 .

[143] X. Ma, N. Zabaras, An efficient Bayesian inference approach to inverse problems based on an adaptive sparse grid collocation method, Inverse Problems 25 (3) (2009) 035013. doi:10.1088/0266-5611/25/3/035013.

[144] Y. M. Marzouk, H. N. Najm, L. A. Rahn, Stochastic spectral methods for efficient Bayesian solution of inverse problems, Journal of Computational Physics 224 (2) (2007) 560-586. doi:10.1016/j.jcp.2006.10.010.

[145] H. Bazargan, M. Christie, A. H. Elsheikh, M. Ahmadi, Surrogate accelerated sampling of reservoir models with complex structures using sparse polynomial chaos expansion, Advances in Water Resources 86 (2015) 385399. doi:10.1016/j.advwatres.2015.09.009.

[146] G. Zhang, D. Lu, M. Ye, M. Gunzburger, C. Webster, An adaptive sparse-grid high-order stochastic collocation method for Bayesian inference in groundwater reactive transport modeling, Water Resources Research 49 (10) (2013) 6871-6892. doi:10.1002/wrcr.20467.

[147] J. Brynjarsdóttir, A. O'Hagan, Learning about physical parameters: The importance of model discrepancy, Inverse Problems 30 (11) (2014) 114007. doi:10.1088/0266-5611/30/11/114007.

[148] K. Beven, J. Freer, Equifinality, data assimilation, and uncertainty estimation in mechanistic modelling of complex environmental systems using the GLUE methodology, Journal of Hydrology 249 (1) (2001) 11-29. doi:10.1016/S0022-1694(01)00421-8.

[149] R. Cooley, S. Christensen, Bias and uncertainty in regression-calibrated models of groundwater flow in heterogeneous media, Advances in Water 
Resources 29 (5) (2006) 639-656. doi:10.1016/j.advwatres.2005.07. 012 .

[150] J. Doherty, D. Welter, A short exploration of structural noise, Water Resources Research 46 (5) (2010) W05525. doi:10.1029/2009WR008377

[151] H. Gupta, M. P. Clark, J. A. Vrugt, G. Abramowitz, M. Ye, Towards a comprehensive assessment of model structural adequacy, Water Resources Research 48 (8) (2012) W08301. doi:10.1029/2011WR011044.

[152] A. Lehikoinen, J. Huttunen, S. Finsterle, M. Kowalsky, J. Kaipio, Dynamic inversion for hydrological process monitoring with electrical resistance tomography under model uncertainties, Water Resources Research 46 (4) (2010) W04513. doi:10.1029/2009WR008470.

[153] D. Erdal, I. Neuweiler, U. Wollschläger, Using a bias aware EnKF to account for unresolved structure in an unsaturated zone model, Water Resources Research 50 (1) (2014) 132-147. doi:10.1002/2012WR013443

[154] D. Calvetti, O. Ernst, E. Somersalo, Dynamic updating of numerical model discrepancy using sequential sampling, Inverse Problems 30 (11) (2014) 114019. doi:10.1088/0266-5611/30/11/114019

[155] Y. Efendiev, T. Hou, W. Luo, Preconditioning Markov chain Monte Carlo simulations using coarse-scale models, SIAM Journal on Scientific Computing 28 (2) (2006) 776-803. doi:10.1137/050628568.

[156] T. Cui, C. Fox, M. O'Sullivan, Bayesian calibration of a large-scale geothermal reservoir model by a new adaptive delayed acceptance Metropolis Hastings algorithm, Water Resources Research 47 (10) (2011) W10521. doi:10.1029/2010WR010352.

[157] L. Josset, V. Demyanov, A. H. Elsheikh, I. Lunati, Accelerating Monte Carlo Markov chains with proxy and error models, Computers \& Geosciences 85 (2015) 38-48. doi:10.1016/j.cageo.2015.07.003. 
[158] T. Smith, A. Sharma, L. Marshall, R. Mehrotra, S. Sisson, Development of

[159] M. Kennedy, A. O'Hagan, Bayesian calibration of computer models, Journal of the Royal Statistical Society: Series B (Statistical Methodology)

[160] D. Higdon, M. Kennedy, J. Cavendish, J. Cafeo, R. D. Ryne, Combining field data and computer simulations for calibration and prediction, SIAM

口 a formal likelihood function for improved Bayesian inference of ephemeral catchments, Water Resources Research 46 (12) (2010) W12551. doi: 10.1029/2010WR009514.

63 (3) (2001) 425-464. doi:10.1111/1467-9868.00294.

Journal on Scientific Computing 26 (2) (2004) 448-466. doi:10.1137/ S1064827503426693

[161] M. Bayarri, J. Berger, R. Paulo, J. Sacks, J. Cafeo, J. Cavendish, C.-H. Lin, J. Tu, A framework for validation of computer models, Technometrics 49 (2007) 138-154. doi:10.1198/004017007000000092

[162] R. Tuo, C. Wu, Efficient calibration for imperfect computer models, The Annals of Statistics 43 (6) (2015) 2331-2352. doi:10.1214/15-A0S1314.

[163] D. Higdon, J. Gattiker, B. Williams, M. Rightley, Computer model calibration using high-dimensional output, Journal of the American

प Statistical Association 103 (482) (2008) 570-583. doi:10.1198/ 016214507000000888

[164] T. Xu, A. Valocchi, A Bayesian approach to improved calibration and prediction of groundwater models with structural error, Water Resources Research 51 (11) (2015) 9290-9311. doi:10.1002/2015WR017912.

[165] R. Tuo, C. Wu, Efficient calibration for imperfect computer models, Annals of Statistics 43(6) (2015) 2331-2352.

[166] R. Tuo, C. Wu, A theoretical framework for calibration in computer models: Parametrization, estimation and convergence properties, SIAM/ASA J. Uncertainty Quantification 4(1) (2016) 767795. 
[167] K. Sargsyan, H. Najm, R. Ghanem, On the statistical calibration of physical models, International Journal of Chemical Kinetics 47 (4) (2015) 246276. doi:10.1002/kin.20906.

[175] T. Ferré, L. Bentley, A. Binley, N. Linde, A. Kemna, K. Singha, K. Holliger, J. A. Huisman, B. Minsley, Critical steps for the continuing advancement of hydrogeophysics, Eos, Transactions American Geophysical Union 90 (23) (2009) 200. 
[177] J. Chen, S. Hubbard, Y. Rubin, Estimating the hydraulic conductivity at

[182] T. Lochbühler, J. Vrugt, M. Sadegh, N. Linde, Summary statistics from training images as prior information in probabilistic inversion, Geophysical Journal International 201(1) (2015) 157-171. doi:10.1093/gji/ggv008

[183] M. Bosch, Lithologic tomography: From plural geophysical data to lithol1390 ogy estimation, Journal of Geophysical Research-Solid Earth 104 (B1) (1999) 749-766. doi:10.1029/1998JB900014. 
[184] M. Bosch, Inference networks in earth models with multiple components and data, in: Integrated Imaging of the Earth: Theory and Ap-

a plications, Geophysical Monograph, Vol. 218, 2016, pp. 29-47. doi: $1395 \quad 10.1002 / 9781118929063 . c h 3$

[185] M. Beaumont, Estimation of population growth or decline in genetically monitored populations, Genetics 164 (3) (2003) 1139-1160. doi:24.

[186] C. Andrieu, G. O. Roberts, The pseudo-marginal approach for efficient Monte Carlo computations, Annals of Statistics 37 (2) (2009) 697-725.

1400 doi:10.1214/07-A0S574.

[187] G. Deligiannidis, A. Doucet, M. K. Pitt, The correlated pseudo-marginal method, arXiv.

[188] A. van der Vaart, Asymptotic Statistics, Cambridge University Press, 2000. 

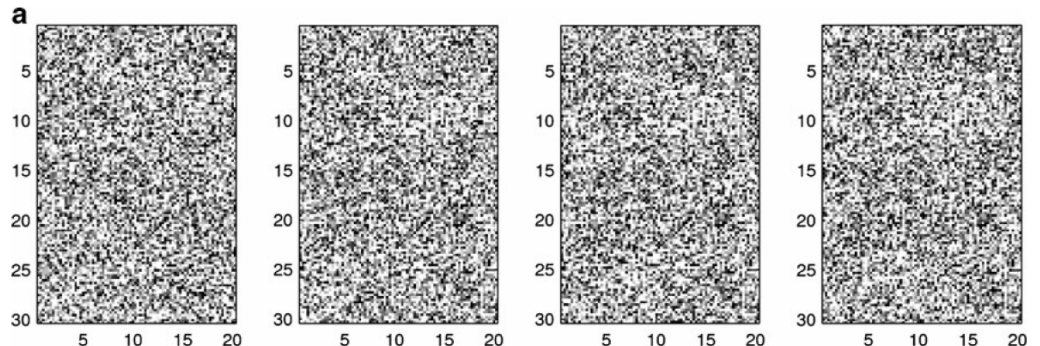

b
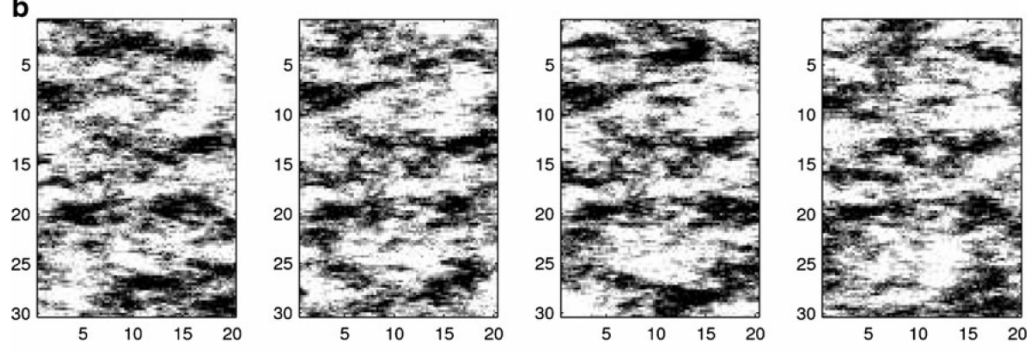

c
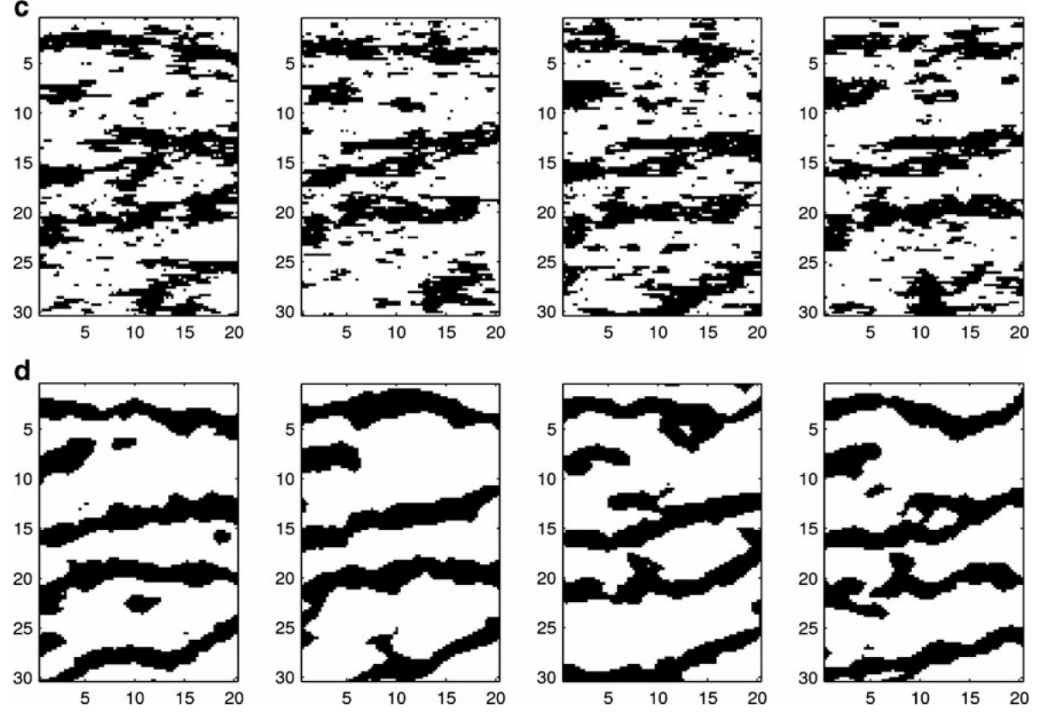

Figure 1: Sampled MCMC posterior realizations based on 800 crosshole first-arrival GPR travel times acquired between the left and the right sides of the model domain. The true subsurface structure (not shown) used to create the data in this synthetic example has channellike features similar to those in (d). The other posterior realizations are based on: (a) a nugget prior model with the correct mean and variance; (b) a Gaussian-random-field prior model with the correct two-point statistics; and (c) the same Gaussian-random-field prior model truncated into a binary field with the correct facies proportions provide realizations that are largely incompatible with the true subsurface structure. From 54 
a)
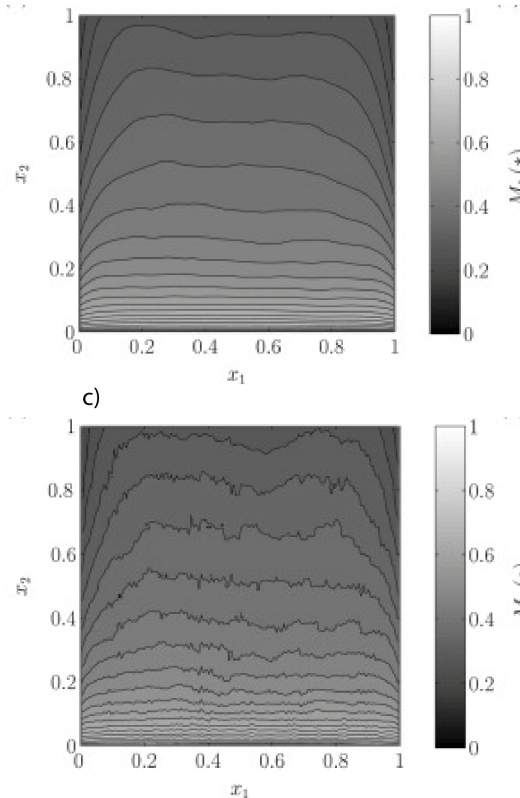

e)

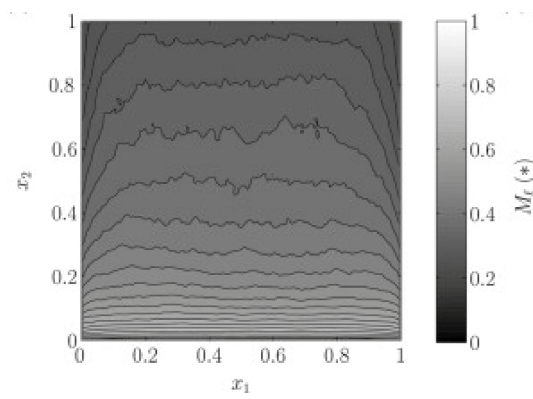

b)

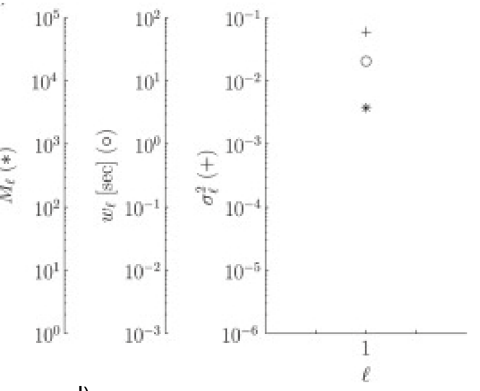

d)

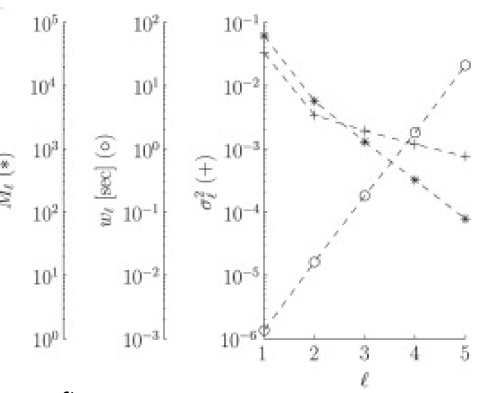

f)

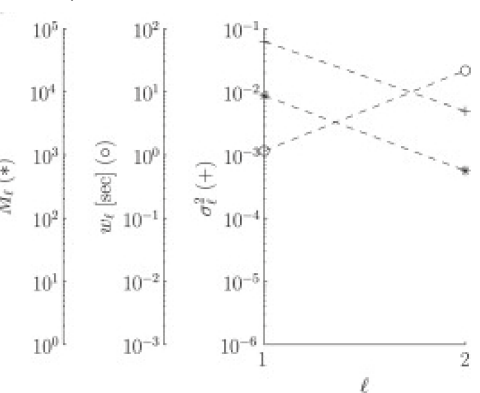

Figure 2: Considering water flooding of a saturated petroleum reservoir, 94 evaluated the performance of MLMC strategies. (a) MC estimation of the mean saturation field at time $t$; and (b) plots showing the number of evaluations at each level, $M_{l}$, the computation time for one evaluation at each level, $w_{l}$, and the variance between levels, $\sigma_{l}^{2}$. Note that there is only one level for the MC case. Corresponding results for MLMC with (c-d) grid and (e-f) solver hierarchy. Note that the mean solutions in (a), (c) and (e) have the same numerical accuracy, while the computational times and the distributions across different levels vary strongly. 


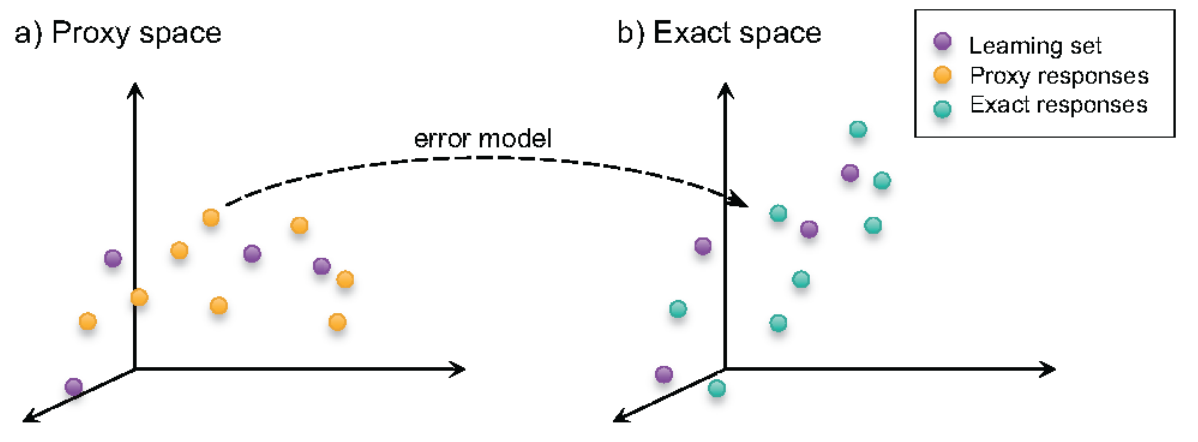

Figure 3: By considering a learning set of contaminant breakthrough curves consisting of proxy responses based on single-phase saline transport simulations "exact" responses obtained using a two-phase solver (purple dots in (a) and (b)), 58 used functional principal components analysis (FPCA) to develop an error model that allows proxy simulations (orange dots in (a)) to be mapped into "exact" responses (blue dots in (b)). Using a learning set based on 20 geostatistical realizations, they demonstrated for a fluvial aquifer with five distinct facies how error-corrected proxy modeling leads to error-corrected predictions that are similar (correlation coefficient of 0.97 ) to the full physics responses. 
(a)
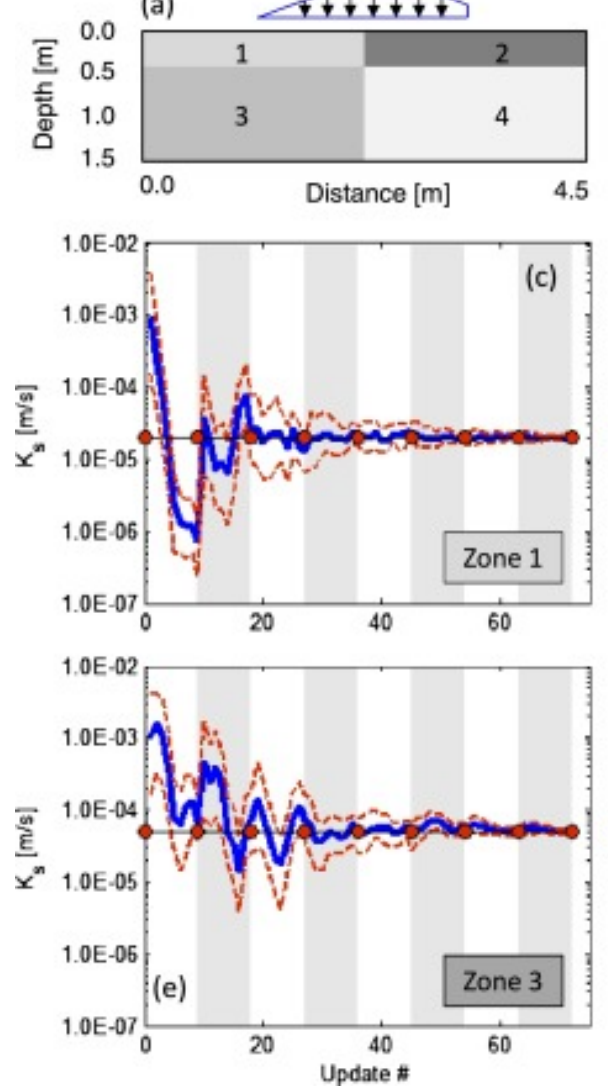

(b)
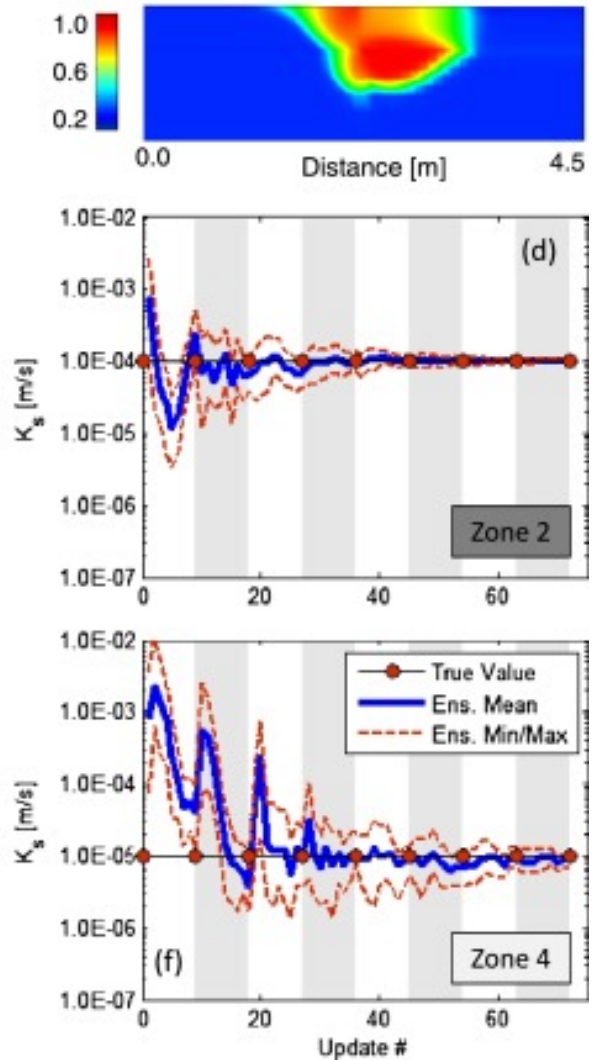

Figure 4: An iterated particle filter method was developed by 114 to infer the hydraulic conductivity of four zones of known geometry given geophysical data. (a) A synthetic infiltration experiment in the vadose zone led to a (b) water plume evolving over time that was sensed by electrical resistivity tomography data under the assumption of a known and perfect petrophysical relationship. (d-f) The inferred hydraulic conductivities converged to the true values. 
(a)

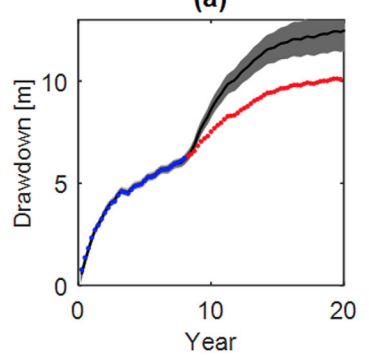

(d)

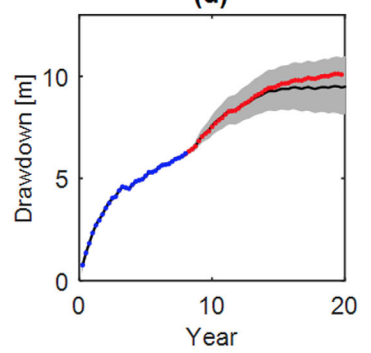

(g)

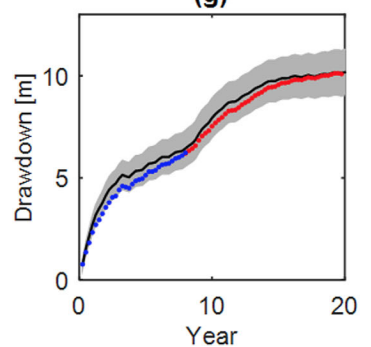

(b)

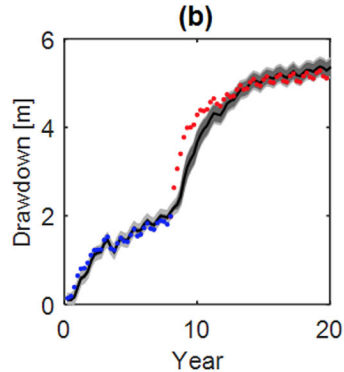

(e)

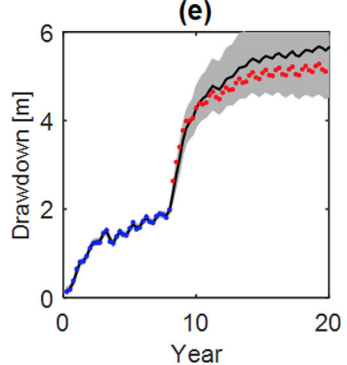

(h)
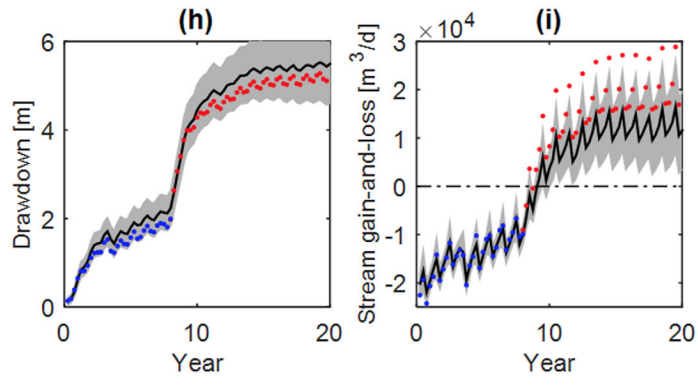

(c)

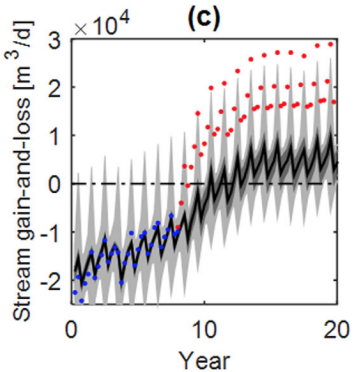

(f)

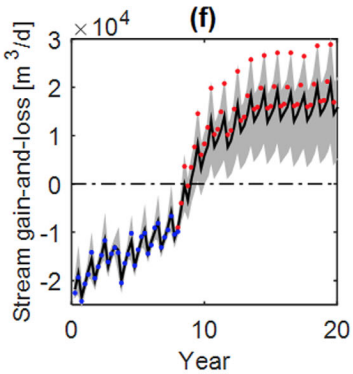

Total Uncertainty

Parameter Uncertainty - Validation data

Mean Prediction

- Calibration observations

Figure 5: 164 considered a synthetic test example involving a "true" 2-D Gaussian hydraulic conductivity field in contact with a river. The inverse problem was parameterized in terms of 12 pilot points. Ignoring model errors caused by this smooth representation leads to biased predictions in terms of (a-b) drawdown at two locations and (c) river-groundwater exchange and unrealistically low uncertainty bounds. By inferring a Gaussian process model describing model errors during the calibration period, the authors obtained (d-f) significantly improved predictions and more realistic uncertainty bounds. Unfortunately, this approach lead to predictions that are unphysical (e.g., not honoring mass constraints). To circumvent this, they considered inversion with a data covariance matrix that include both the observational and the previously inferred model errors. (g-i) The corresponding predictions based on the resulting inversion model are physically-consistent and the bias is low. 

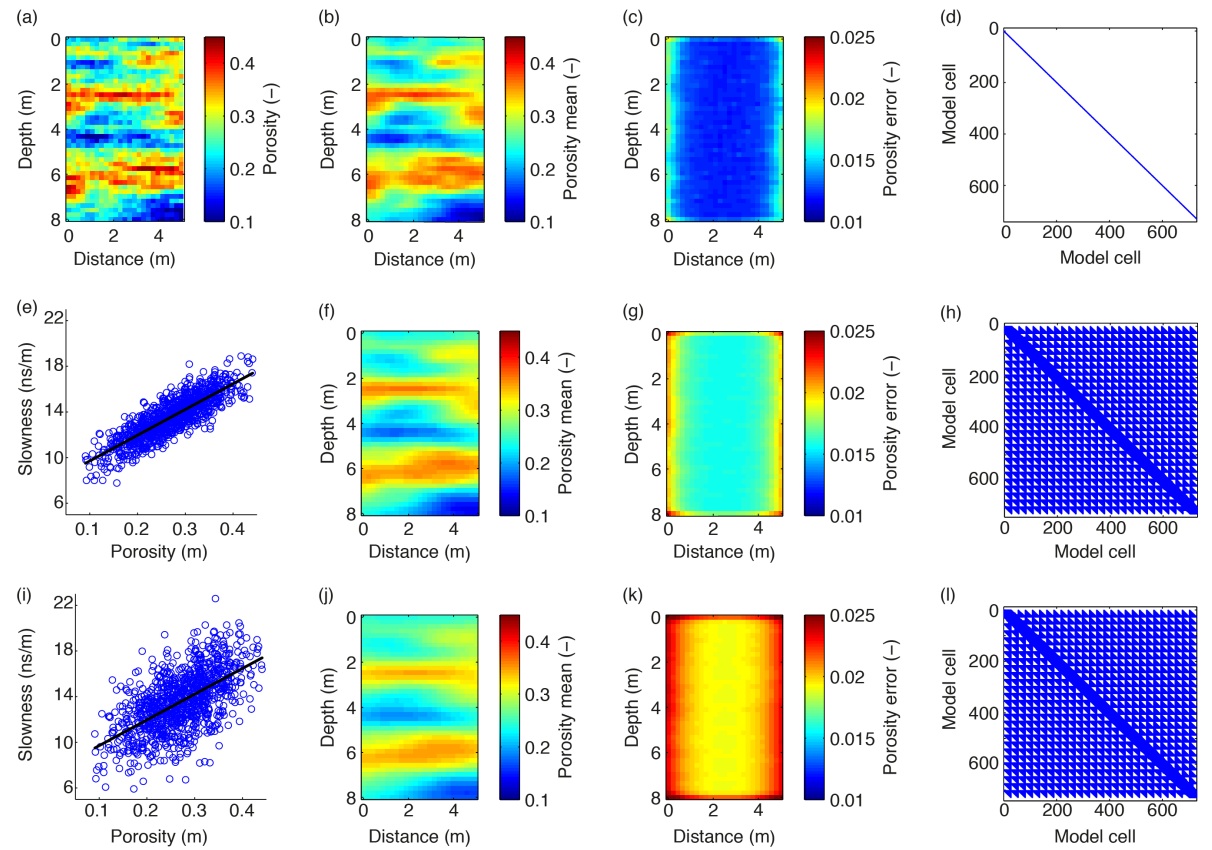

Figure 6: Synthetic example of porosity inference from crosshole GPR travel time data under assumptions of linear theory, a known Gaussian-random-field model, and uncorrelated data and petrophysical errors. (a) True porosity field, (b) inferred mean model, (c) standard deviation and (d) structure of the data covariance matrix under the assumption of a perfect petrophysical relationship (black line in (e)). (e) Strong petrophysical relationship and resulting (f) mean model, (g) standard deviation and (h) structure of the data covariance matrix. (j-l) corresponding results for a (i) rather strong petrophysical relationship. 